\title{
Data Stream Evolution Diagnosis Using Recursive Wavelet Density Estimators
}

\author{
EDGAR S. GARCÍA TREVIÑO, MUHAMMAD ZAID HAMEED, and JAVIER A. BARRIA, \\ Imperial College London
}

\begin{abstract}
Data streams are a new class of data that is becoming pervasively important in a wide range of applications, ranging from sensor networks, environmental monitoring to finance. In this article, we propose a novel framework for the online diagnosis of evolution of multidimensional streaming data that incorporates Recursive Wavelet Density Estimators into the context of Velocity Density Estimation. In the proposed framework changes in streaming data are characterized by the use of local and global evolution coefficients. In addition, we propose for the analysis of changes in the correlation structure of the data a recursive implementation of the Pearson correlation coefficient using exponential discounting. Two visualization tools, namely temporal and spatial velocity profiles, are extended in the context of the proposed framework. These are the three main advantages of the proposed method over previous approaches: (1) the memory storage required is minimal and independent of any window size; (2) it has a significantly lower computational complexity; and (3) it makes possible the fast diagnosis of data evolution at all dimensions and at relevant combinations of dimensions with only one pass of the data. With the help of the four examples, we show the framework's relevance in a change detection context and its potential capability for real world applications.
\end{abstract}

CCS Concepts: • Information systems $\rightarrow$ Data stream mining; • Computing methodologies $\rightarrow$ Online learning settings;

Additional Key Words and Phrases: Data streams mining, data stream evolution diagnosis, velocity density estimation, incremental statistics

ACM Reference format:

Edgar S. García Treviño, Muhammad Zaid Hameed, and Javier A. Barria. 2018. Data Stream Evolution Diagnosis Using Recursive Wavelet Density Estimators. ACM Trans. Knowl. Discov. Data. 12, 1, Article 14 (January 2018), 28 pages.

https://doi.org/10.1145/3106369

\section{INTRODUCTION}

The expansion of the Internet and the progress of hardware technology have enabled the emergence of a new class of data, the so-called data streams. Data streams are temporal data objects that are becoming pervasively and increasingly important in a wide variety of applications, e.g., sensor networks, network monitoring, transaction log analysis, financial tickers, and web usage.

This work of M. Z. Hameed is supported by an Imperial College London President's PhD Scholarship. Authors' addresses: E. S. G. Treviño, M. Z. Hameed, and J. A. Barria, Department of Electrical and Electronic Engineering, Imperial College London, London SW7 2BT, United Kingdom; emails: edgar.garcia-trevino08@alumni.imperial.ac.uk, \{muhammad.hameed13, j.barria\}@imperial.ac.uk.

This work is licensed under a Creative Commons Attribution International 4.0 License.

2018 Copyright is held by the owner/author(s).

ACM 1556-4681/2018/01-ART14

https://doi.org/10.1145/3106369

ACM Transactions on Knowledge Discovery from Data, Vol. 12, No. 1, Article 14. Publication date: January 2018. 
In such contexts, data arrive in a continuous online time-varying fashion at a rapid rate and it is not feasible to store it or to exchange it in order to apply traditional database queries.

This new class of data specifically leads to two underlying challenges (Aggarwal 2007). Since the volume of the data is continuously increasing at a rapid rate, suitable algorithms should process each data item only once, that is, they should work with only one pass of the data. Moreover, newly developed algorithms should take into account time dependent relations as well as the evolution of the underlying data (García-Treviño and Barria 2014).

Data stream mining issues have recently attracted the attention of the machine learning and pattern recognition communities. According to the data mining problem they address, available methods can be categorized into the following: clustering (e.g., Rodrigues et al. (2008) and Dai et al. (2006)), classification (e.g., Aggarwal and Yu (2007)), indexing (e.g., Faloutsos (2004) and Gopalkrishnan (2008)), frequent pattern mining (e.g., Cormode and Hadjieleftheriou (2009)), change detection (e.g., Wang et al. (2003), Kuncheva (2013), Liu et al. (2013), and Desobry et al. (2005)), summarisation, (including sketching, load shedding and synopsis construction) (e.g., Cormode and Garofalakis (2007)), and forecasting (e.g., Yang et al. (2006)).

In the context of streaming data, evolution diagnosis focuses on the investigation and characterization of changes that occur over time in the trends of a given data stream due to changes in the underlying phenomena under observation (Aggarwal 2005): consider a data stream to be a sequence $\left\langle\mathrm{X}_{1}, \mathrm{X}_{2}, \cdots\right\rangle$, where each $\mathrm{X}_{\mathrm{t}}$ is a multidimensional data point generated by some probability density function $f_{t}$ and each $\mathbf{X}_{\mathbf{t}}$ is independent from previous data points. The problem of data evolution can be stated as the characterization of the difference between a specific underlying feature of the data at time $t_{1}$, and a reference feature of the same data at some other time $t_{2}$. Note that since this difference is also a function, its characterization involves not only its overall quantification, but more importantly, it also considers the description of how it is changing over time. Data evolution problem might seem quite similar to that of change detection. However, the key difference is that while change detection focuses mostly on the quantification of the amount of the change, evolution diagnosis tries in addition to characterize changes, and in that sense, provides additional and valuable insights on how the generative process of the data evolves over time. In the remainder of this article, the data point $\mathrm{X}_{\mathrm{t}}$ at time $t$ is characterized by its density function $f_{t}$.

In this article, we propose a novel evolution diagnosis framework for data streams which extends the concept of velocity density estimation developed in Aggarwal (2005); to measure the rate of change of data concentration at a given spatial location over a user-defined time horizon. Aggarwal's framework was originally introduced within the context of an offline Kernel Density Estimation (KDE). Here, we extend this framework with a Recursive Wavelet Density Estimator (RWDE), which is optimized for online applications. Furthermore, instead of formulating the velocity density estimation in terms of sliding windows, the proposed framework makes use of an exponential discounting strategy. Also, in Aggarwal (2005), to estimate velocity density profiles and in general to quantify data evolution two types of densities are considered, namely a forward density estimate and a reverse density estimate. In the framework proposed in this article, the concepts of reverse and forward density do not apply and instead we use a pair of density estimates related to different exponential discounting/updating strategies for the coefficients of each estimator. The last important difference is that in the proposed framework velocity densities are formulated in a separate way for each dimension. This capability not only allows the localized diagnosis of data evolution in each dimension but it also provides the basis for the detection of particular dimensions or combinations of dimensions in the data that are relevant for a given data change.

In the framework here proposed, the characterization of changes in streaming data is based on concepts such as evolution coefficient and correlation between evolution coefficients. An evolution 
coefficient is an indicator of the level of significance of data changes at each particular time stamp. Here a high level of evolution is associated to significant changes of data concentrations at various spatial locations over a particular time horizon. The correlation coefficient (CC) between evolution coefficients corresponding to different dimensions captures changes in the correlation structure of the data. Specifically, we propose a recursive implementation of the Pearson correlation coefficient using exponential discounting. Additionally, two visual profiles, i.e., temporal velocity profiles and spatial velocity profiles that were proposed in Aggarwal (2005) are also extended to visualize our results. Moreover, the proposed framework has some important advantages with respect to the approach reported in Aggarwal (2005): (1) It requires a significant lower amount of memory; (2) the proposed algorithms are computationally less complex; and (3) for high dimensional data, it allows the diagnosis of data evolution separately for each dimension with only one pass of the data.

The framework reported in this article is very relevant when designing data stream mining algorithms, as it considers the time element in the analysis. For example, the inclusion of the time element is crucial to the following three aspects of the analysis (Silva et al. 2013): (i) In time-aware clustering (Chen and Tu 2007; Kranen et al. 2011), which focuses on how the age of the objects (clusters) affect the clustering process, the proposed framework can provide insights on the evolution of each cluster and when its corresponding definition needs to be updated. (ii) In the outlier-evolution dilemma (Wang et al. 2003; Cao et al. 2006), that focuses on correctly distinguishing between outliers and cluster evolution, the proposed framework can enhance the detection of relevant underlying changes in the probability distribution of the data and adapt the clusters accordingly; or to identify objects that had been deemed as outliers but are in fact part of a new emerging cluster. (iii) In the so-called clustering tracking (Oliveira and Gama 2012; Spiliopoulou et al. 2006), where it is envisaged that data stream clustering algorithms should be able to monitor the dynamic behavior of the clusters over time, the proposed framework can enhance the understanding of the cluster evolution, providing insights on the nature of cluster change evolution; e.g., when a cluster is disappearing, when its members are migrating to other clusters, when a new emerging cluster reflects a new profile of objects or when a new cluster consist of old objects whose characteristics have evolved.

The rest of the article is organized as follows. In Section 2, theoretical background regarding RWDE is briefly reviewed. In Section 3, the proposed data stream evolution diagnosis framework is presented. Section 4 includes the empirical evaluation of the framework. Final remarks are discussed in Section 5.

\section{THEORETICAL BACKGROUND}

\subsection{Recursive Wavelet Density Estimator Overview}

2.1.1 Batch Wavelet Density Estimator (WDE). The WDE falls into the class of orthogonal series estimators, originally introduced in Céncov (1962). Here, an unknown square integrable density function can be expressed as a convergent series of orthogonal basis functions $f(x)=\sum_{j} b_{j} \varphi_{j}(x)$, where $\left\{\varphi_{j}\right\}$ is a complete orthonormal system of basis functions in $L^{2}(\mathbb{R}), b_{j}$ is the coefficient of the $j$ th basis function and $j \in \mathcal{J} \subset \mathbb{Z}$ is an appropriate set of indices. In this context, if $X_{1}, X_{2}, \ldots, X_{n}$ are the realizations of a random variable $X$, then the coefficient $b_{j}$ can be expressed as the expectation: $b_{j}=\left\langle f, \varphi_{j}\right\rangle=\int \varphi_{j}(x) f(x) d x=E\left[\varphi_{j}(X)\right]$ with $j \in \mathcal{J}$. Consequently, the $j$ th series coefficient in an orthogonal series estimator can be approximated by $\hat{b}_{j}=\frac{1}{n} \sum_{i=0}^{n} \varphi_{j}\left(X_{i}\right)$.

Within the wavelet framework the density can be represented as an orthogonal series of scaling functions $\phi$ and wavelet functions $\psi$. Since in data streams applications we are constrained by 
computational restrictions, in this work we consider the simplest WDE, which is a linear estimator defined by

$$
\hat{f}(x)=\sum_{k} \hat{c}_{j_{0}, k} \phi_{j_{0}, k}(x)
$$

where $\phi_{j_{0}, k}(x)=2^{j_{0} / 2} \phi\left(2^{j_{0}} x-k\right)$ is the scaling function associated with the base resolution $2^{-j_{0}}$ with $j_{0} \in \mathbb{Z}$ denoting the scale and with the index $k \in \mathcal{K} \subset \mathbb{Z}$. In order to simplify notation, in this article, we focus our attention on scaling function filters whose support at resolution $2^{-j_{0}}=0$ is $2 N_{\phi_{0}}-1$ with $N_{\phi_{0}}$ denoting the order of the filter. The well-known Daubechies and Symmlets wavelet families include scaling functions with these characteristics. Assuming that each $X_{i}$ is normalized to take only values within the interval $[0,1]$, and considering $\left[2^{j_{0}} k, 2^{j_{0}}\left(k+2 N_{\phi_{0}}-1\right)\right]$ to be the support of $\phi_{j_{0}, k}$, then $\mathcal{K}=\left\{-\left(2 N_{\phi_{0}}-2\right), \ldots, 0, \ldots,\left(2^{j_{0}}-1\right)\right\}$ (see García-Treviño and Barria (2012) for more details).

In (1), coefficients $\hat{c}_{j_{0}, k}$ are estimated according to Vidakovic (1999):

$$
\hat{c}_{j_{0}, k}=\frac{1}{n} \sum_{i=1}^{n} \phi_{j_{0}, k}\left(X_{i}\right) .
$$

It has been shown in Vidakovic (1999) that the estimator $\hat{f}(x)$ is suboptimal. However, since in the proposed framework the interest is placed on the fast evaluation of the relative difference between densities rather than in their precise estimation, then the prompt detection of relative changes in the density is very relevant.

2.1.2 Extension to Higher Dimensions. The estimator described above can be easily extended to higher dimensions by considering multidimensional multiresolution analysis and their corresponding multidimensional wavelets. In this case, if we consider $\mathbf{X}_{i}=\left(X_{i}^{1}, X_{i}^{2}, \ldots, X_{i}^{m}\right) \in \mathbb{R}^{m}$ to be the realizations of a multidimensional random variable $\mathrm{X}$ and using $\mathbf{x}=\left(x_{1}, x_{2}, \ldots, x_{m}\right) \in \mathbb{R}^{m}$ then the $m$-dimensional density can be expressed as

$$
\hat{f}(\mathbf{x})=\sum_{\mathbf{k}} \hat{c}_{j_{0}, \mathbf{k}} \Phi_{j_{0}, \mathbf{k}}(\mathbf{x})
$$

where, $\mathbf{k}=\left(k_{1}, k_{2}, \ldots, k_{m}\right) \in \mathbb{Z}^{m}$ and $j_{0} \in \mathbb{Z}$. Following (2), the $m$-dimensional extension for the approximation of the scaling coefficients is given by

$$
\hat{c}_{j_{0}, \mathrm{k}}=\frac{1}{n} \sum_{i=1}^{n} \Phi_{j_{0}, \mathrm{k}}\left(\mathbf{X}_{i}\right),
$$

where the $m$-dimensional basis function is defined by $\Phi_{j_{0}, \mathrm{k}}(\mathbf{x})=\bigotimes_{d=1}^{m} \phi_{j_{0}, k}\left(x_{d}\right)$, where the symbol $\bigotimes$ denotes tensor product. Note that we follow the concept of separable multiresolution approximations (Ogden 1997) in which $m$-dimensional scaling functions are defined as the tensor product of $m$ one-dimensional scaling functions.

2.1.3 Recursive WDE. For the online implementation of the WDE we use the recursive estimator proposed in Caudle and Wegman (2009), that we refer as to RWDE and which originally comprises two stages. The initial stage is an offline stage in which an initial estimate of the density is obtained using the batch WDE of (1) and (2). In the second stage, the estimator coefficients $\hat{c}_{j_{0}, k}(t)$ are recursively updated online as new data items arrive according to the following equation:

$$
\hat{c}_{j_{0}, k}(t)=(1-\theta) \hat{c}_{j_{0}, k}(t-1)+(\theta) \phi_{j_{0}, k}\left(X_{t}\right)
$$

where $X_{t}$, the newest data item available, denotes the realization of a random variable $X$ at time $t$. Since coefficients $\hat{c}_{j_{0}, k}$ are estimated recursively each time a new data item arrives, the temporal 
index $t$ is included. Hence, (5) defines an exponential discounting strategy for the estimator coefficients where the parameter $\theta, 1 \geq \theta \geq 0$, controls the emphasis assigned to new data with respect to older one. ${ }^{1}$ In order to make faster the proposed framework, we include the selective evaluation of coefficients reported in García-Treviño and Barria (2012) for (5). In this way, prior to the updating of $\hat{c}_{j_{0}, k}(t)$, we check if $X_{t}$ falls within the interval $\left[2^{j_{0}} k, 2^{j_{0}}\left(k+2 N_{\phi_{0}}-1\right)\right]$, which is the support of $\phi_{j_{0}, k}$. If $X_{t}$ is inside this interval then we evaluate $(\theta) \phi_{j_{0}, k}\left(X_{t}\right)$, otherwise we make zero that part of the equation.

\subsection{The Velocity Density Estimation Framework}

The velocity density concept in the context of KDE and sliding windows, which was introduced in Aggarwal (2005), is based on the intuitive idea that high levels of evolution in a given data stream are associated to changes in relative data concentrations at some given spatial locations. The concept of velocity density relies on the difference between two probability densities, each of them associated to a particular temporal smoothing/weighting strategy for the data covered by the sliding window $\left(t-h_{t}, t\right)$, where $h_{t}$ defines the length of the temporal window. For this purpose, a forward time slice density estimate and a reverse time slice density estimate are employed. While the former is related to the probability density for all data items covered by the sliding window giving a higher importance to new data, the latter relies on computing the probability density of the data emphasizing the importance of the old data inside the window.

Note that in Aggarwal (2005) the probability densities are estimated using KDE that require the same number of base functions (i.e., Gaussian functions) as the number of data items used for the estimation. Also, if $h_{t}$ is chosen to be large, not only the amount of memory required will increase, but also the computational burden of the algorithm will be higher. Moreover, the crucial disadvantage of obtaining evolution coefficients using the approach reported in Aggarwal (2005) is the fact that this process requires the evaluation of the density at given points distributed in a multidimensional grid. Hence, as the number of dimensions increases so do the number of evaluation points needed but in an exponential way. If we assume, for instance, that a given density is characterized by evaluating 10 points equally distributed along the support of the density in each dimension, then the number of resulting evaluation points for a data stream of dimension $m$ is $10^{m}$. This makes the method of Aggarwal (2005) unpractical for analyzing evolution of multidimensional data streams. Furthermore, for the case of multidimensional data, the evaluation of the amount of evolution at each particular dimension involves scanning the data more than once. The above disadvantages prevent the potential deployment of the above framework in real world applications.

In order to overcome the above issues and in order to provide an intuitive user-friendly data stream evolution framework, in this article, we propose the estimation of velocity density using a different density estimator and a rather different data discounting strategy.

\section{PROPOSED FRAMEWORK}

In this section, we reformulate the velocity density estimation framework proposed in Aggarwal (2005) by replacing the sliding window approach by an exponential discounting strategy for old data. We use the RWDE estimator as it does not require the storage of past data items in memory (as it will be further discussed in Section 3.3.6). In addition to this, the proposed discounting strategy employs a fixed number of basis functions, independent of the number of data items used for the estimation; and not only allows the evaluation of data evolution at each dimension separately,

\footnotetext{
${ }^{1}$ In order to find a simple direct relation between $\theta$ and the window size parameter used in the context of sliding windows for the weight assigned to the previous value of the coefficients $c_{j_{0}, k}$, in this article, we use $(1-\theta)$ instead of $\theta$.
} 


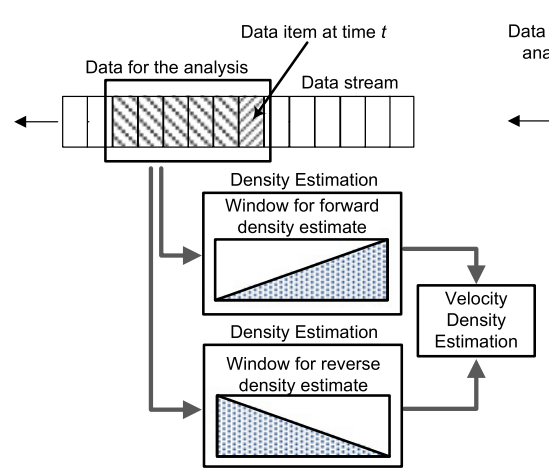

(a)

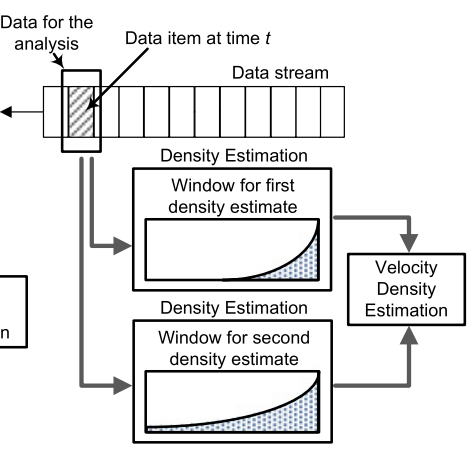

(b)

Fig. 1. Windows used in the evolution diagnosis frameworks. (a) Framework reported in Aggarwal (2005). (b) Framework proposed in this article.

but it also provides a radically different and computationally less expensive framework for the estimation of local evolution coefficients (LECs) and global evolution coefficients (GECs).

It is important to point out a fundamental theoretical difference between the proposed framework and the approach reported in Aggarwal (2005). The evolution diagnosis framework of Aggarwal (2005) is based on velocity densities which are constructed by comparing two probability density functions that are estimated using one sliding window but applying different temporal smoothing strategies. Hence, the key idea in Aggarwal (2005) is the use of a spatiotemporal kernel function that is a time-factored version of the spatial kernel, traditionally used in the KDE context. In contrast, the density estimation framework we propose, relies on the use of two recursive exponential windows-applying different exponential discounting strategies for old data-which only require the last data item available to update the density estimates at a given time stamp.

An underlying advantage of the proposed technique is that it requires constant space for the computation of the densities, independent of any window size. For example, if we are interested in quantifying long-term changes in data, this can be analyzed without increasing memory storage. In contrast, using the framework reported in Aggarwal (2005), the size of the sliding window and consequently, the amount of memory required and the computational complexity of the algorithm will increase.

The two approaches for velocity density estimation are depicted in Figure 1. Note that the two main differences between the methods are the following: (1) the data used for the estimation of the density; and (2) the type of window used for the estimation. While the method shown in Figure 1(a) requires all the data that falls inside the sliding window, the proposed framework, shown in Figure 1(b), only requires the last data item. Regarding the window employed by the weighting strategy, the approach reported in Aggarwal (2005) uses a linear one (see Figure 1(a)), whereas in the proposed approach (Figure 1(b)), it is an exponentially decaying one.

\subsection{Proposed Velocity Density Framework}

Let's consider a multivariate data stream similar to the one defined in Section 2.1.2, whose multidimensional data items at time $t, \mathbf{X}_{t}=\left(X_{t}^{1}, X_{t}^{2}, \ldots, X_{t}^{m}\right) \in \mathbb{R}^{m}$, are assumed to be the realizations of a multidimensional random variable $\mathrm{X}$.

3.1.1 Density Estimation. In our framework two types of velocity densities are employed, both of them relying on the RWDE estimation approach reviewed in Section 2.1. The characterization 
of evolution is based on using a pair of one-dimensional probability density estimates of the form:

$$
\hat{f}_{\theta}^{d}\left(x_{d}, t\right)=\sum_{k} \hat{c}_{j_{0}, k}^{d}(t) \phi_{j_{0}, k}\left(x_{d}\right),
$$

where $\theta$ defines a given discounting strategy and with the coefficients of the estimator recursively updated each time a new data item is available according to

$$
\hat{c}_{j_{0}, k}^{d}(t)=(1-\theta) \hat{c}_{j_{0}, k}^{d}(t-1)+\theta \phi_{j_{0}, k}\left(X_{t}^{d}\right),
$$

where $X_{t}^{d}$ refers to the last data item available at time $t$ and dimension $d$. Here, $k \in \mathcal{K}=\left\{-\left(2 N_{\phi_{0}}-\right.\right.$ $\left.2), \ldots, 0, \ldots,\left(2^{j_{0}}-1\right)\right\}$.

The visualization of evolution dynamics is based on two-dimensional velocity densities relying on two-dimensional densities expressed as

$$
\hat{f}_{\theta}^{d_{a}, d_{b}}\left(\mathbf{x}^{d_{a}, d_{b}}, t\right)=\sum_{\mathbf{k}} \hat{c}_{j_{0}, \mathbf{k}}^{d_{a}, d_{b}} \Phi_{j_{0}, \mathbf{k}}\left(\mathbf{x}^{d_{a}, d_{b}}\right),
$$

where the coefficients $c_{j_{0}, \mathbf{k}}^{d_{a}, d_{b}}$ are calculated according to

$$
\hat{c}_{j_{0}, \mathbf{k}}^{d_{a}, d_{b}}(t)=(1-\theta) \hat{c}_{j_{0}, \mathbf{k}}^{d_{a}, d_{b}}(t-1)+\theta \Phi_{j_{0}, \mathbf{k}}\left(X_{t}^{d_{a}, d_{b}}\right) .
$$

Note that the two-dimensional basis functions $\Phi_{j_{0}, \mathrm{k}}$ are obtained following the procedure explained in Section 2.1.2, and in this sense they are defined as

$$
\Phi_{j_{0}, \mathbf{k}}\left(X_{t}^{d_{a}, d_{b}}\right)=\phi_{j_{0}, k}\left(X_{t}^{d_{a}}\right) \otimes \phi_{j_{0}, k}\left(X_{t}^{d_{b}}\right),
$$

where $d_{a}$ and $d_{b}$ indicate the dimensions selected for the analysis.

Note that in (6) to (9), we introduce the temporal variable $t$ to both, density estimates and coefficients, to indicate their time dependency. Also different values of $\theta$ reflect different discounting strategies for old data in estimator.

Note also that the RWDE algorithm proposed in Caudle and Wegman (2009) comprises two stages namely, an offline and an online stage. However, since in data streaming applications the estimation of an initial density is not feasible, in our implementation of RWDE, we use only its online stage, initializing the estimator coefficients to zero, that is, $\hat{c}_{j_{0}, k}^{d}(0)=0 \forall k \in \mathcal{K}$. Here, the evaluation of (8) and (6) requires, for all dimensions, the same set of $P$ points $\beta=\left\{b_{P}\right\}_{p \in 1,2, \ldots, P}$, with $b_{P} \in[0,1]$. Algorithms 1 and 2 summarize the two stage approach of the RWDE algorithm for the one-dimensional case. Algorithm 1 shows the pseudo code of the updating of the estimators coefficients stage, and Algorithm 2 shows the pseudo code of the evaluation of the density stage.

Since the velocity density framework is based on the quantification of the difference between two densities, in the proposed approach a pair of RWDE with different values for $\theta$ is required.

3.1.2 The Selection of $\theta$. The procedure for the selection of $\theta$ involves making the weighting value of a particular past coefficient equal to a user defined parameter $c_{\min }$ related to the desired weighting value for coefficient $\hat{c}_{j_{0}, k}(t-w)$, where $w$ is an hypothetical window length, with $0<$ $c_{\text {min }}<1, w>1$ and $w \in \mathbb{N}$. To this end, first consider that (5) can expanded in the following way:

$$
\begin{aligned}
\hat{c}_{j_{0}, k}(t)= & (1-\theta)^{t} \hat{c}_{j_{0}, k}(0) \\
& +(1-\theta)^{t-1} \theta \hat{c}_{j_{0}, k}(1)+(1-\theta)^{t-2} \theta \hat{c}_{j_{0}, k}(2) \\
& +\ldots(1-\theta) \theta \hat{c}_{j_{0}, k}(t-1)+\theta \phi_{j_{0}, k}(x(t)) .
\end{aligned}
$$




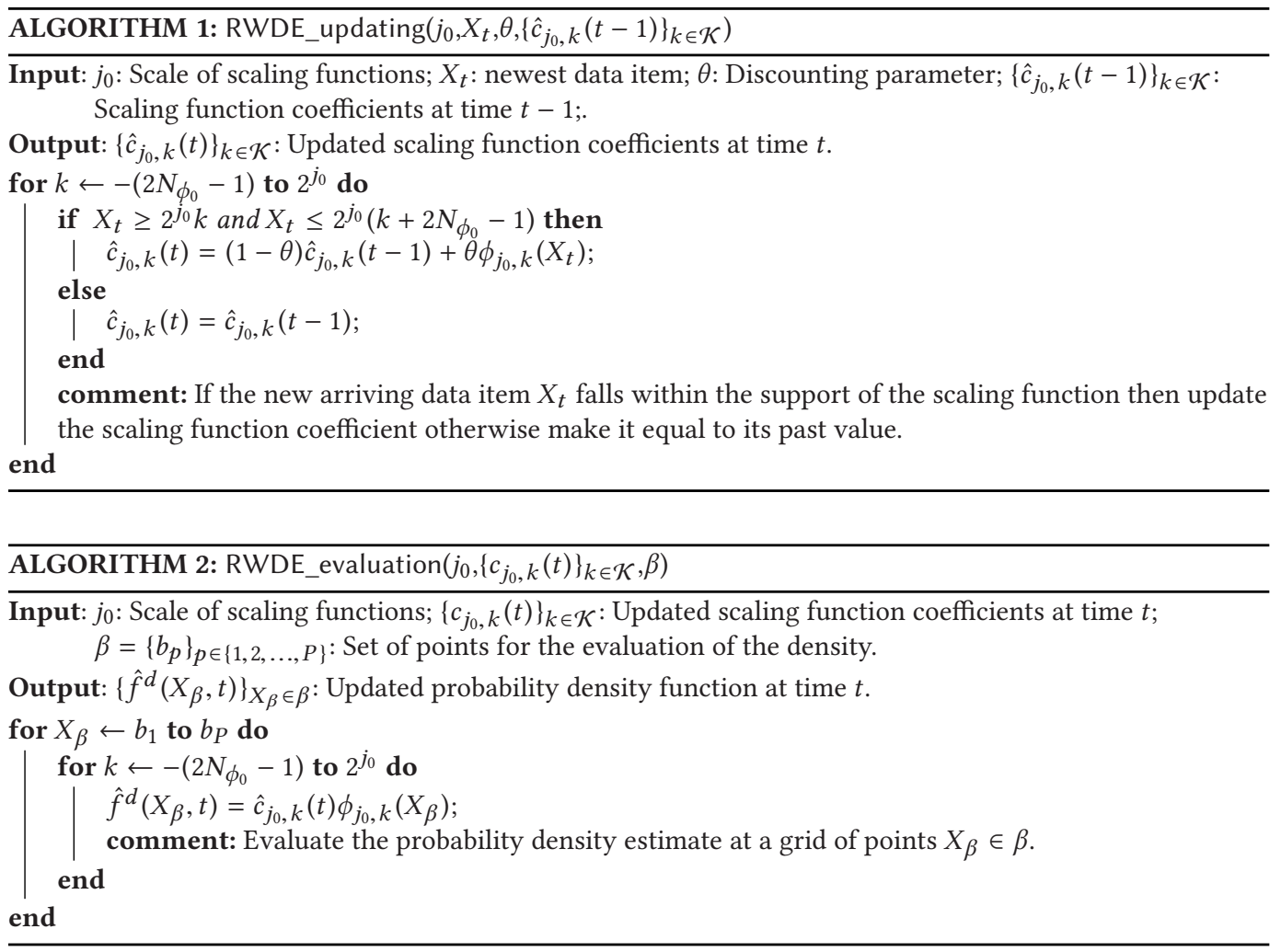

According to (11), the weight assigned to the coefficient $\hat{c}_{j_{0}, k}(t-w)$, that is the last coefficient covered by an hypothetical window of length $w$, is $(1-\theta)^{t-(t-w)} \theta$. Taking into account this, $\theta$ can be estimated from the following equation:

$$
\begin{aligned}
(1-\theta)^{t-(t-w)} \theta & =c_{\min }(1-\theta) \theta \\
(1-\theta)^{w-1} & =c_{\min } \\
\theta & =1-\left(c_{\min }\right)^{1 /(w-1)} .
\end{aligned}
$$

Specifically, for normalization, in (12), we consider that the weighting value for the coefficient $\hat{c}_{j_{0}, k}(t-w)$ should be equal to $c_{\text {min }}$ times the weighting value of coefficient $\hat{c}_{j_{0}, k}(t-1)$ that is $(1-\theta) \theta$. In order to establish a simple relation between $\theta$ and the window size $w$, in this article, we use $c_{\text {min }}=e^{-1}=0.3679$, which means that the weighting value corresponding to the coefficient obtained at time $t-w$, the oldest element in the hypothetical window, has a contribution of $36.79 \%$. By choosing this particular value for $c_{\min }$ then $\theta$ becomes approximately equal to $1 / w$.

In the proposed velocity density framework, we require two densities with different discounting strategies related to two different window lengths, $w_{1}$ and $w_{2}$. We use the notation $\theta_{w_{1}}$ and $\theta_{w_{2}}$ to refer the discounting parameters associated to $w_{1}$ and $w_{2}$, respectively. Note that $w_{2}>w_{1}$ and $w_{1}, w_{2} \in \mathbb{N}$ are user defined quantities whose values depend on the desired time horizon for the analysis.

In order to be able to benchmark the results to the ones obtained using the sliding window approach reported in Aggarwal (2005), if no explicitly mentioned otherwise, we select $w_{1}=w_{2} / 2$. As it is discussed in Section 4.1, the proposed framework offers more flexibility in the selection of 
$w_{1}$ and $w_{2}$. This means that, as long as $w_{2}>w_{1}$, there is no restriction in the values selected for $w_{1}$ and $w_{2}$.

3.1.3 Velocity Density Calculation. Two different strategies for the estimation of velocity densities are here presented.

Velocity Density from Densities (VDD). The first strategy for the obtention of velocity densities is based on estimating the difference between two different density estimates of the data directly from the densities. We call this method Velocity Density from Densities (VDD). In this case, by using the one-dimensional densities of (6), the one dimensional velocity density $V_{\left(\theta_{w_{1}}, \theta_{w_{2}}\right)}^{d}\left(X^{d}, t\right)$ at spatial location $X^{d}$ and time $t$ for dimension $d$ is defined by

$$
V_{\left(\theta_{w_{1}}, \theta_{w_{2}}\right)}^{d}\left(X^{d}, t\right)=\frac{\hat{f}_{\theta_{w_{1}}}^{d}(x, t)-\hat{f}_{\theta_{w_{2}}}^{d}(x, t)}{w_{2}-w_{1}},
$$

where $\theta_{w_{1}}$ and $\theta_{w_{2}}$ are, as we said before, different exponential discounting strategies, and $w_{1}$ and $w_{2}$ represent the window lengths used for the estimation.

Velocity Density from Estimator Coefficients (VDC). In the context of RWDE, the way a given density vary over time is directly related to the way its corresponding parameters (i.e., wavelet coefficients) are changing. We call this strategy Velocity Density from Coefficients (VDC).

This novel strategy for velocity density has the advantage of a reduced computational burden since it is directly computed from the estimator coefficients, avoiding the evaluation, at every time stamp $t$, of the density (using either (8) or (6)) in a set of coordinates $\beta$. This alternative velocity can be expressed, for the one-dimensional case, as

$$
\hat{V}_{\left(\theta_{w_{1}}, \theta_{w_{2}}\right)}^{d}(k, t)=\frac{\hat{\mathbf{c}}_{\theta_{w_{1}}}^{d}(k, t)-\hat{\mathbf{c}}_{\theta_{w_{2}}}^{d}(k, t)}{w_{2}-w_{1}}
$$

where $\hat{\mathbf{c}}_{\theta_{w_{x}}}^{d}(k, t)=\left[\hat{c}_{j_{0},-2 N_{\phi_{0}-1}}^{d}(t), \ldots, c_{j_{0}, 2^{j_{0}}}^{d}(t)\right]$ is a vector containing the $\left(2^{j_{0}}+2 N_{\phi_{0}}-2\right)$ estimator coefficients using the discounting strategy defined by $w_{x}$. Note here that the velocity presented in (14) is a discrete function since $k$ is a discrete set of translation parameters for the scaling function $\phi_{j_{0}, k}$.

\subsection{Visualization of Changes: Visual Profiles}

In this section, we extended the visualization tool proposed in Aggarwal (2005) into the RWDE context. The visualization of change is based on the VDDs approach since on the VDCs approach the set of coordinates $\beta$ is fixed and does not allow the visualization of a particular spatial location of the data with a higher level of detail.

3.2.1 Temporal Velocity Profiles (TVPs). The temporal velocity profile is defined as the global overview of the velocity density at different spatial points for a given specific time (Aggarwal 2005). This profile is a surface plot of the velocity density using a user defined grid of spatial locations or coordinates among two dimensions. Since this visualization tool is useful for two-dimensional data, we require two-dimensional velocity densities of the form:

$$
V_{\left(\theta_{w_{1}}, \theta_{w_{2}}\right)}^{d_{a}, d_{b}}\left(X^{d_{a}, d_{b}}, t\right)=\frac{\hat{f}_{\theta_{w_{1}}}^{d_{a}, d_{b}}\left(\mathbf{x}^{d_{a}, d_{b}}, t\right)-\hat{f}_{\theta_{w_{2}}}^{d_{a}, d_{b}}\left(\mathbf{x}^{d_{a}, d_{b}}, t\right)}{w_{2}-w_{1}},
$$



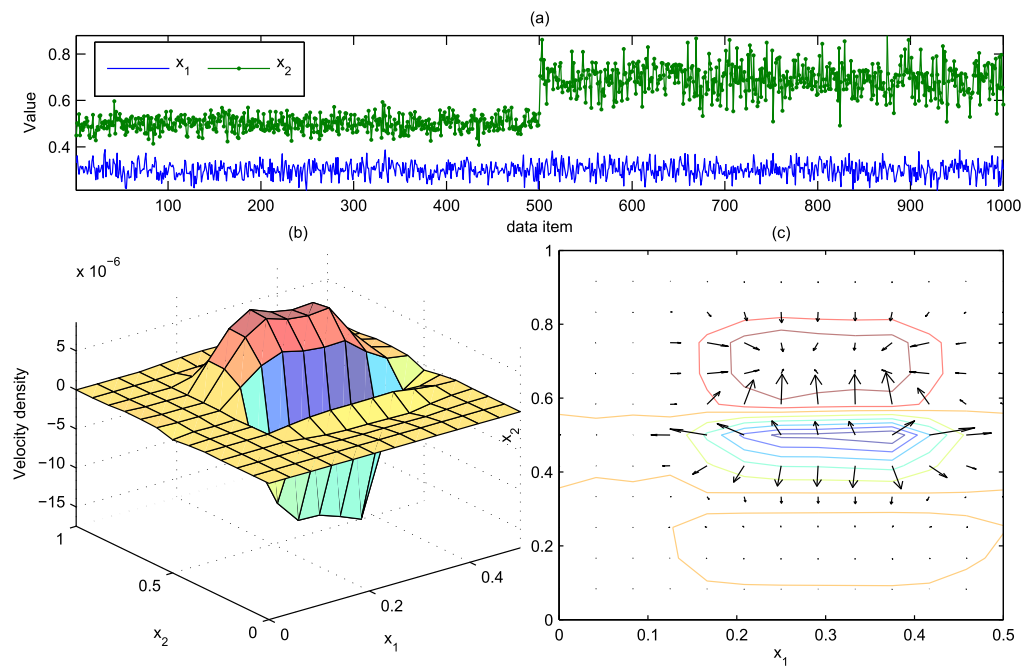

Fig. 2. A two-dimensional data stream (a) and its corresponding TVP (b) and SVP (c).

where $d_{a}$ and $d_{b}$ are the two dimensions of the data relevant for the analysis and $X^{d_{a}, d_{b}}$ is the spatial location in which the velocity density is evaluated. Note that $\hat{f}_{\theta_{w_{1}}}^{d_{a}, d_{b}}\left(\mathbf{x}^{d_{a}, d_{b}}, t\right)$ and $\hat{f}_{\theta_{w_{2}}}^{d_{a}, d_{b}}\left(\mathbf{x}^{d_{a}, d_{b}}, t\right)$ are two-dimensional densities.

For the construction of TVPs a discretized version of the velocity density is required. This discrete two-dimensional VDD is obtained by evaluating (15) in a set of coordinates $\beta=\left\{b_{P}\right\}_{p \in 1,2, \ldots, P}$ along each dimension. For the set of coordinates of $P$ points, each of the two densities used in the construction of the velocity density are evaluated.

3.2.2 Spatial Velocity Profiles (SVPS). This visualization profile is defined as the spatial overview of the reorganizations that are taken place in the data density at specific spatial points (Aggarwal 2005). The SVPs allow the user to easily observe the associated directions of all those changes that are taking place in the data. Intuitively, when data is shifting from one spatial location to another the source of the shift shows a reduction in the probability density, whereas the destination of the shift shows an increment. Therefore, there is an increasing gradient from the source to the destination of the shift. The construction of this profile first estimates the gradient at given grid of spatial locations, and then plots the corresponding velocity vectors as arrows at those locations.

For the construction of SVPs, we use a set of coordinates $\beta$ along each dimension with respect to which the gradient of the velocity density will be evaluated. Then for each spatial location $X^{d_{a}, d_{b}}$ in that grid the velocity gradient along the $i$ th dimension is defined by

$$
\Delta v_{i}\left(X^{d_{a}, d_{b}}, t\right)=\lim _{\epsilon \rightarrow 0} \frac{V_{\left(\theta_{w_{1}}, \theta_{w_{2}}\right)}^{d_{a}, d_{b}}\left(X^{d_{a}, d_{b}}+\overline{\epsilon_{i}}, t\right)-V_{\left(\theta_{w_{1}}, \theta_{w_{2}}\right)}^{d_{a}, d_{b}}\left(X^{d_{a}, d_{b}}, t\right)}{\epsilon},
$$

where $\overline{\epsilon_{i}}=\epsilon \cdot \overline{e_{i}}$ is an $\epsilon$-perturbation along the $i$ th dimension and with $\overline{e_{i}}$ denoting a unit vector. In Figure 2, we plot the corresponding TVPs and SVPs for an example two-dimensional data stream. It can be seen from TVP that velocity density is positive around spatial location of 0.7 in second dimension $\left(x_{2}\right)$ with respect to first dimension $\left(x_{1}\right)$ as reference, as greater number of data stream points close to this spatial location have arrived at the end of time horizon used for estimation. On the other hand, velocity density is negative around spatial location 0.5 in second dimension 
(with first dimension as reference), where more data stream points arrived in the beginning of the interval. From the SVP, it is clear that data stream has shifted in second dimension $\left(x_{2}\right)$ from spatial location of 0.5 to 0.7 with first dimension $\left(x_{1}\right)$ remaining unchanged. Here, at each spatial location, the gradient vector represents the direction and magnitude of shift in data stream.

\subsection{Characterizing Data Evolution}

3.3.1 Data Evolution Coefficient. In order to quantitatively address the level of significance of changes in streaming data, we propose the evaluation of both local and global data evolution coefficients (Aggarwal 2005) using VDC. LECs are related to single dimensions of the data stream, whereas the GECs consider the entire sets of dimensions.

The proposed framework relies on the observation that the amount of evolution of a given data stream at a given time instant $t$ is directly proportional to the sum of all changes in the estimator coefficients. Since each of these coefficients is related to a particular spatial location, then changes in data concentrations at given location directly produce variations in the value of the corresponding coefficients. In this sense, a high level of evolution is associated to significant changes among all these coefficients.

An important advantage of the proposed framework is that since velocity densities are separately computed for each dimension, then evolution coefficients can also be obtained in the same manner. For the case of high dimensional data, this is an important capability that allows the straightforward identification of dimensions in data in which data evolution is relevant and dimensions in which data is not significantly changing. We define the LEC corresponding to the dimension $d$ of the data as

$$
E_{\left(\theta_{w_{1}}, \theta_{w_{2}}\right)}^{d}(t)=\left(w_{2}-w_{1}\right) \sum_{k}\left|\hat{V}_{\left(\theta_{w_{1}}, \theta_{w_{2}}\right)}^{d}(k, t)\right|
$$

Note here that by using (17), we not only can quantify in which particular dimension the data is more significantly evolving, but also we can find a subset of the most relevant dimensions for that underlying data change.

If a general overview of the evolution is needed then a GEC can be used. As one representative instantiation, we propose in this article estimating the GEC by averaging the evolution coefficients at each dimension $E_{\left(\theta_{w_{1}}, \theta_{w_{2}}\right)}^{d}(t)$ according to

$$
\tilde{E}_{\left(\theta_{w_{1}}, \theta_{w_{2}}\right)}(t)=\frac{1}{m} \sum_{d=1}^{m} E_{\left(\theta_{w_{1}}, \theta_{w_{2}}\right)}^{d}(t)
$$

3.3.2 Correlation Between Evolution Coefficients. Since the proposed framework is based on the estimation of evolution at each dimension, the correlation structure of the data stream can be assessed by finding the correlation between evolution coefficients corresponding to different dimensions. We propose to compute the CC between two one-dimensional streams using an exponential discounting method. Inspired by the method to compute the CC for sliding windows introduced in Wang and Wang (2003) and Rodrigues et al. (2008), in this article, we propose an Exponentially Weighted Moving Correlation Coefficient (EWMCC) algorithm that can be recursively computed using only the newest data item available.

For simplicity, let us make $a(t)=E_{\left(\theta_{w_{1}}, \theta_{w_{2}}\right)}^{a}(t)$ and $b(t)=E_{\left(\theta_{w_{1}}, \theta_{w_{2}}\right)}^{b}(t)$. Here, $a(t)$ and $b(t)$ can be seen as streams of evolution coefficients for two different dimensions. Then the EWMCC between streams of evolution coefficients corresponding to dimensions $a$ and $b$ using an exponential 
window defined by $\alpha$ is expressed as

$$
\widehat{C C}_{(a, b)}(t)=\frac{C(t)-D(t) \bar{A}(t) \bar{B}(t)}{\sqrt{A_{2}(t)-D(t)(\bar{A}(t))^{2}} \sqrt{B_{2}(t)-D(t)(\bar{B}(t))^{2}}},
$$

with

$$
\begin{aligned}
& \bar{A}(t)=\left(1-D(t)^{-1}\right) \bar{A}(t-1)+D(t)^{-1} a(t) ; \\
& \bar{B}(t)=\left(1-D(t)^{-1}\right) \bar{B}(t-1)+D(t)^{-1} b(t) ; \\
& C(t)=(1-\alpha) C(t-1)+a(t) b(t) ; \\
& A_{2}(t)=(1-\alpha) A_{2}(t-1)+a(t)^{2} ; \\
& B_{2}(t)=(1-\alpha) B_{2}(t-1)+b(t)^{2} ; \\
& D(t)=(1-\alpha) D(t-1)+1 .
\end{aligned}
$$

Note here that all the elements of (19) are also computed recursively. Here, $\bar{A}(t)$ and $\bar{B}(t)$ are the recursive version of the mean of $a(t)$ and $b(t)$, respectively. $A_{2}(t)$ and $B_{2}(t)$ are the recursive versions of the sum of $a(t)^{2}$ and $b(t)^{2}$, respectively. $C(t)$ is the recursive implementation of the product of $a(t)$ and $b(t)$, whereas $D(t)$ is a recursive variable related to the number of data items effectively included inside the exponential window. For initialization, we set $\bar{A}(1)=a(1)$, $\bar{B}(1)=b(1), C(1)=a(1) b(1), A_{2}(1)=a(1)^{2}, B_{2}(1)=b(1)^{2}$, and $D(1)=1$. In (19), $\alpha=1 / w_{\alpha}$ is an user defined value that depends on to the length of the time window $w_{\alpha}$ used for the analysis. The pseudo code for the proposed EWMCC is shown in Algorithm 3.

ALGORITHM 3: $\operatorname{EWMCC}(a(t), b(t), \gamma(t-1))$;

Input: $a(t)$ : Evolution coefficient at time $t$ for the first dimension of the analysis; $b(t)$ : Evolution coefficient at time $t$ for the second dimension of the analysis; $\gamma(t-1)=\{\bar{A}(t-1), \bar{B}(t-1)$,

$\left.A_{2}(t-1), B_{2}(t-1), C(t-1), D(t-1)\right\}$ : Set of recursive parameters for the estimation of the correlation coefficient.

Output: $\widehat{\operatorname{corr}}_{(a, b)}(t)$ : Correlation coefficient between streams of evolution coefficients $a$ and $b$ at time $t$; $\gamma(t)$ : Updated set of recursive parameters.

$\bar{A}(t)=\left(1-D(t)^{-1}\right) \bar{A}(t-1)+D(t)^{-1} a(t) ;$

$\bar{B}(t)=\left(1-D(t)^{-1}\right) \bar{B}(t-1)+D(t)^{-1} b(t) ;$

$C(t)=(1-\alpha) C(t-1)+a(t) b(t)$

$A_{2}(t)=(1-\alpha) A_{2}(t-1)+a(t)^{2} ;$

$B_{2}(t)=(1-\alpha) B_{2}(t-1)+b(t)^{2}$.

$D(t)=(1-\alpha) D(t-1)+1$.

$\widehat{\operatorname{corr}}_{(a, b)}(t)=\frac{C(t)-D(t) \bar{A}(t) \bar{B}(t)}{\sqrt{A_{2}(t)-D(t)(\bar{A}(t))^{2}} \sqrt{\bar{B}_{2}(t)-D(t)(\bar{B}(t))^{2}}}$

comment: Evaluate the EWMCC between two one-dimensional streams of evolution coefficients $a$ and $b$ at time $t$.

In Figure 3, we plot an example two-dimensional data stream and the corresponding CC reported by the proposed EWMCC algorithm and the sliding window approach of Wang and Wang (2003) at every time stamp. We can observe from Figure 3, that results obtained using EWMCC are similar to results computed using a sliding window approach, which means that EWMCC is able to capture, with some arbitrary precision defined by the parameter $w_{\alpha}$, the changing correlation of data stream $a$.

3.3.3 Finding Relevant 2D Projections of Data. The plotting of TVPs and SPVs is relevant for all those pairs of dimensions (2D projections) in which the correlation structure of the data is more significantly evolving. By considering that in a $m$-dimensional data stream there are $N_{c}$ 2-combinations of the form $\left(\begin{array}{c}m \\ 2\end{array}\right)$, then to compute all of them using (19) $m$ recursive means 


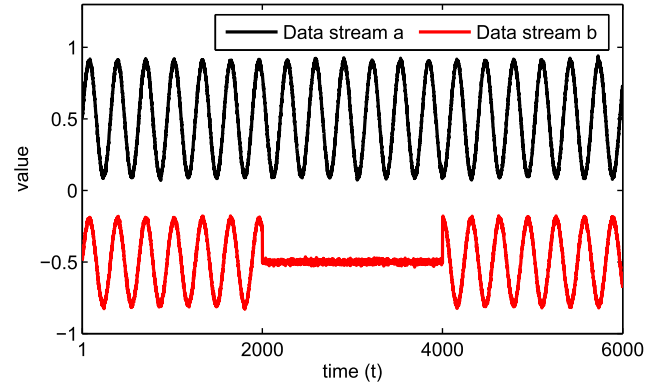

(a)

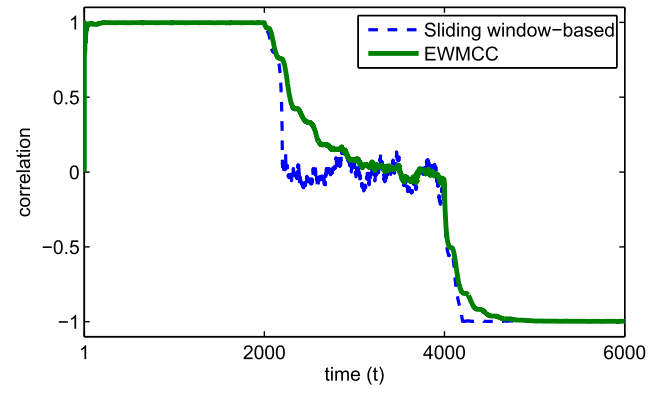

(c)

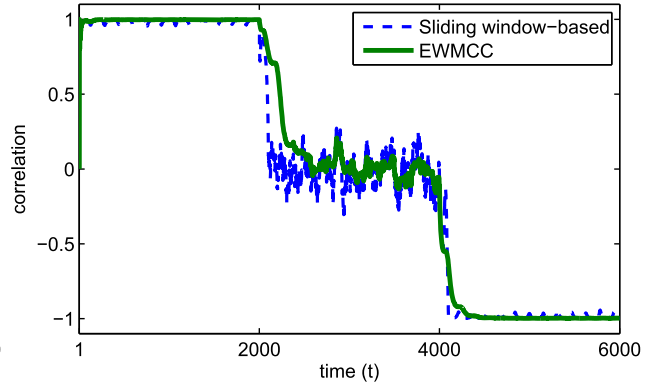

(b)

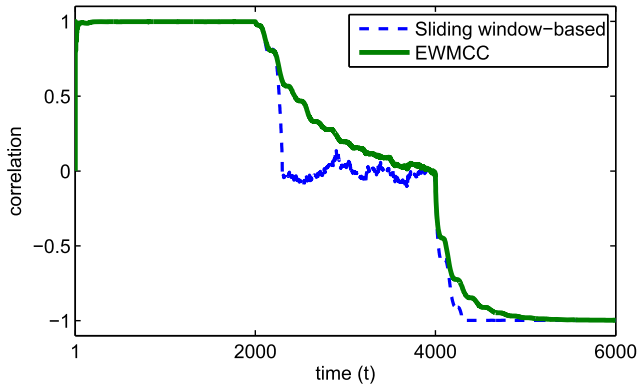

(d)

Fig. 3. Evaluation of techniques for the online computing of CC. (a) Two example data streams selected for the analysis; (b) CC and EWMCC using $w=w_{\alpha}=100$; (c) CC and EWMCC using $w=w_{\alpha}=200$; (d) CC and EWMCC using $w=w_{\alpha}=300$.

(either $\bar{A}(t)$ or $\bar{B}(t)), m$ recursive sums of squared one-dimensional data streams (either $a(t)^{2}$ or $\left.b(t)^{2}\right)$ as well as $N_{c}$ recursive products $a(t) b(t)$ are required to be stored. Note that only one recursive variable $D(t)$ is required since it is common to all dimensions. Then in total $2 m+N_{c}+1$ recursive variables need to be maintained. For instance, if $m=100$ there are $N_{c}=4,925$ combinations of pairs of dimensions for which the storage of $2(100)+4,925+1=5,126$ variables is required. Furthermore, the updating of each of these recursive variables involves only two simple multiplications.

3.3.4 Computational Complexity. The complexities of the proposed VDD and VDC are directly related to the complexity of the one-dimensional RWDE in which they both rely on. RWDE in turn involves, for each data item $X_{i}$, the evaluation of the scaling function $\phi_{j_{0}, k}\left(X_{i}\right)$ for each of the $N_{b}=\left(2^{j_{0}}+2 N_{\phi_{0}}-2\right)$ scaling functions employed using $j_{0}$. The evaluation of $\phi_{j_{0}, k}\left(X_{i}\right)$ is performed using the so-called Daubechies-Lagarias Algorithm (see Vidakovic (1999) for more details) where two variables are involved, i.e., the order of the filter $N_{\phi_{0}}$ and the precision of the algorithm $r$ (in this article, we consider $r=9$ ). Specifically, the complexity of evaluating $\phi_{j_{0}, k}\left(X_{i}\right)$ for a single scaling function is $O\left(r\left(2 N_{\phi_{0}}-1\right)^{3}\right)$. In this sense, $O\left(r N_{b}\left(2 N_{\phi_{0}}-1\right)^{3}\right)$ is the complexity of updating all the estimator coefficients at every time stamp. According to this, the complexity of the one-dimensional VDC is $O\left(2 r N_{b}\left(2 N_{\phi_{0}}-1\right)^{3}\right)$, while the complexity of the $m$-dimensional VDC is $O\left(2 m r N_{b}\left(2 N_{\phi_{0}}-1\right)^{3}\right)$. Since VDD, in addition to the updating of the estimator coefficients also involves the evaluation of the density in a set of $P$ points, its complexity for the one-dimensional case is given by $O\left(2 r\left(N_{b}+P\right)\left(2 N_{\phi_{0}}-1\right)^{3}\right)$, whereas for the $m$-dimensional it is $O\left(2 m r\left(N_{b}+P\right)\left(2 N_{\phi_{0}}-1\right)^{3}\right)$. 
Table 1. Complexity Comparison of Velocity Density Methods

\begin{tabular}{cccccc|cccccc}
\hline$m$ & $h_{t}$ & $P$ & VDC $^{*}$ & VDD $^{*}$ & VDKDE & $m$ & $h_{t}$ & $P$ & VDC $^{*}$ & VDD $^{*}$ & VDKDE \\
\hline 1 & 1,000 & 10 & $1.4 \mathrm{e}+05$ & $2.0 \mathrm{e}+05$ & $2.0 \mathrm{e}+04$ & 1 & 1,000 & 20 & $1.4 \mathrm{e}+05$ & $2.6 \mathrm{e}+05$ & $4.0 \mathrm{e}+04$ \\
10 & 1,000 & 10 & $1.4 \mathrm{e}+06$ & $2.0 \mathrm{e}+06$ & $2.0 \mathrm{e}+14$ & 10 & 1,000 & 20 & $1.4 \mathrm{e}+06$ & $2.6 \mathrm{e}+06$ & $2.0 \mathrm{e}+17$ \\
50 & 1,000 & 10 & $6.8 \mathrm{e}+06$ & $9.9 \mathrm{e}+06$ & $1.0 \mathrm{e}+55$ & 50 & 1,000 & 20 & $6.8 \mathrm{e}+06$ & $1.3 \mathrm{e}+07$ & $1.1 \mathrm{e}+70$ \\
\hline 1 & 5,000 & 10 & $1.4 \mathrm{e}+05$ & $2.0 \mathrm{e}+05$ & $1.0 \mathrm{e}+05$ & 1 & 5,000 & 20 & $1.4 \mathrm{e}+05$ & $2.6 \mathrm{e}+05$ & $2.0 \mathrm{e}+05$ \\
10 & 5,000 & 10 & $1.4 \mathrm{e}+06$ & $2.0 \mathrm{e}+06$ & $1.0 \mathrm{e}+15$ & 10 & 5,000 & 20 & $1.4 \mathrm{e}+06$ & $2.6 \mathrm{e}+06$ & $1.0 \mathrm{e}+18$ \\
50 & 5,000 & 10 & $6.8 \mathrm{e}+06$ & $9.9 \mathrm{e}+06$ & $5.0 \mathrm{e}+55$ & 50 & 5,000 & 20 & $6.8 \mathrm{e}+06$ & $1.3 \mathrm{e}+07$ & $5.6 \mathrm{e}+70$ \\
\hline 1 & 10,000 & 10 & $1.4 \mathrm{e}+05$ & $2.0 \mathrm{e}+05$ & $2.0 \mathrm{e}+05$ & 1 & 10,000 & 20 & $1.4 \mathrm{e}+05$ & $2.6 \mathrm{e}+05$ & $4.0 \mathrm{e}+05$ \\
10 & 10,000 & 10 & $1.4 \mathrm{e}+06$ & $2.0 \mathrm{e}+06$ & $2.0 \mathrm{e}+15$ & 10 & 10,000 & 20 & $1.4 \mathrm{e}+06$ & $2.6 \mathrm{e}+06$ & $2.0 \mathrm{e}+18$ \\
50 & 10,000 & 10 & $6.8 \mathrm{e}+06$ & $9.9 \mathrm{e}+06$ & $1.0 \mathrm{e}+56$ & 50 & 10,000 & 20 & $6.8 \mathrm{e}+06$ & $1.3 \mathrm{e}+07$ & $1.1 \mathrm{e}+71$ \\
\hline
\end{tabular}

${ }^{*}$ Using $r=9, N_{\phi_{0}}=4, j_{0}=4$.

3.3.5 Computational Complexity Comparison. In this section, we theoretically compare the computational complexity of the proposed VDD and VDC methods with the velocity density framework proposed in Aggarwal (2005), which we refer to as VDKDE. For the one-dimensional case, the complexity of VDKDE is $O\left(2 h_{t} P\right)$, where $h_{t}$ is the length of the sliding window. For the $m$-dimensional case, it is $O\left(2 m h_{t} P^{m}\right)$. Note here that the number of points in which the densities are evaluated depends on the number of dimensions $m$.

For comparison, in Table 1, we provide the number of multiplications required to compute velocity densities using VDC, VDD, and VDKDE when the number of dimensions of the data $m$, the size of the window for the analysis $h_{t}$ and the number of $P$ points in which each density is evaluated are varied.

The most important observation from Table 1 is that, since the proposed VDC and VDD algorithms are independent of the window size $h_{t}$, they are significantly less complex than VDKDE, in particular for cases involving higher dimensions.

3.3.6 Space Complexity. The space complexity of the proposed VDD and VDC algorithms is both constant and independent of any window size. Specifically, both algorithms require the storage of the latest evolution coefficient at each dimension plus the coefficients of the density estimators employed, which can be expressed as $2 m N_{b}$, where $N_{b}$ is the number of scaling functions used in RWDE. In contrast, the space complexity of the VDKDE framework is $2 h_{t}$, which directly depends on the window size $h_{t}$. In Table 2, we show the space complexity of the proposed and the VDKDE methods for different window sizes and for different dimensions. The main observation from this table is the fact that the constant space complexity of the VDD and VDC algorithms makes them suitable for the analysis of large amounts of data.

\section{EMPIRICAL EVALUATION AND EXAMPLES}

The evaluation of the proposed algorithm consists of the following five parts ${ }^{2}$ : (i) selection of parameters and evolution coefficients; (ii) computational complexity assessment; (iii) change detection and evolution diagnosis example, and (iv) three examples on the application of the framework to real world applications.

\footnotetext{
${ }^{2}$ The computer system used to generate the results reported in this section was an Intel i5 2500k with 16GB of RAM, running Linux Ubuntu 11.04 and the simulation environment was MATLAB R2010b.
} 
Table 2. Space Complexity of Velocity Density Methods

\begin{tabular}{cccccc}
\hline & & \multicolumn{3}{c}{ VDC and VDD } & VDKDE \\
$m$ & $h_{t}$ & $j_{0}=3$ & $j_{0}=4$ & $j_{0}=5$ & \\
\hline 1 & $1 \mathrm{e}+04$ & $2.9 \mathrm{e}+01$ & $4.5 \mathrm{e}+01$ & $7.7 \mathrm{e}+01$ & $2.0 \mathrm{e}+04$ \\
10 & $1 \mathrm{e}+04$ & $2.9 \mathrm{e}+02$ & $4.5 \mathrm{e}+02$ & $7.7 \mathrm{e}+02$ & $2.0 \mathrm{e}+04$ \\
100 & $1 \mathrm{e}+04$ & $2.9 \mathrm{e}+03$ & $4.5 \mathrm{e}+03$ & $7.7 \mathrm{e}+03$ & $2.0 \mathrm{e}+04$ \\
\hline 1 & $1 \mathrm{e}+06$ & $2.9 \mathrm{e}+01$ & $4.5 \mathrm{e}+01$ & $7.7 \mathrm{e}+01$ & $2.0 \mathrm{e}+06$ \\
10 & $1 \mathrm{e}+06$ & $2.9 \mathrm{e}+02$ & $4.5 \mathrm{e}+02$ & $7.7 \mathrm{e}+02$ & $2.0 \mathrm{e}+06$ \\
100 & $1 \mathrm{e}+06$ & $2.9 \mathrm{e}+03$ & $4.5 \mathrm{e}+03$ & $7.7 \mathrm{e}+03$ & $2.0 \mathrm{e}+06$ \\
\hline 1 & $1 \mathrm{e}+08$ & $2.9 \mathrm{e}+01$ & $4.5 \mathrm{e}+01$ & $7.7 \mathrm{e}+01$ & $2.0 \mathrm{e}+08$ \\
10 & $1 \mathrm{e}+08$ & $2.9 \mathrm{e}+02$ & $4.5 \mathrm{e}+02$ & $7.7 \mathrm{e}+02$ & $2.0 \mathrm{e}+08$ \\
100 & $1 \mathrm{e}+08$ & $2.9 \mathrm{e}+03$ & $4.5 \mathrm{e}+03$ & $7.7 \mathrm{e}+03$ & $2.0 \mathrm{e}+08$ \\
\hline
\end{tabular}

${ }^{*}$ Using $r=9, N_{\phi_{0}}=4$.

\subsection{Selection of Parameters and Evolution Coefficients}

In this section, we compare the evolution coefficients as they convey the amount of variation information contained in the velocity density in a single number. As an illustrative example, we consider the one-dimensional synthetic data stream depicted in Figure 4(a) that presents a shift in mean. Specifically, this data stream was generated by drawing a random number from a Gaussian distribution with fixed variance $\sigma^{2}=0.001$ and mean that changes over time (from the 1 to the 1,000 data item $\mu=0.5$, from the 2,000 data item to $3,000 \mu=0.7$, while from the 1,001 data item to the $2,000 \mu$ changes from 0.5 to 0.7 increasing 0.0002 at each data item).

In Figure 4(f), it is shown that the proposed framework allows the use of different wavelet families, all of which can capture the evolution of a data stream. We use wavelets from the Symlets family, since they are the common choice for WDE (Vidakovic 1999), as they are the "least asymmetric" compactly supported orthogonal wavelets.

The order of the scaling function $N_{\phi_{j_{0}}}$ also impacts the resulting evolution coefficient as it is shown in Figure 4(e). From the six orders evaluated only the wavelet Sym1 (of order 1), presents a degraded behavior. It is important to highlight that $N_{\phi_{j_{0}}}$ is directly related to approximation capabilities of the scaling function and in the relevant WDE literature (Vidakovic 1999), $N_{\phi_{j_{0}}}=4$ has been suggested to use for good approximation.

The pair of window sizes $w_{1}$ and $w_{2}$ are application specific parameters since they both define the data considered for the analysis. Specifically, $w 1$ defines the subset of the most recent data that is compared with the reference old data defined by $w_{2}$. In Figure $4(\mathrm{~b})$ and (c), we evaluate the effect of modifying the difference $w_{2}-w_{1}$ (maintaining $w_{1}$ fixed and only changing $w_{2}$ ) and the effect of maintaining constant the difference $w_{2}-w_{1}$. Note that as $w_{1}$ and $w_{2}$ or their difference increase the magnitude of their corresponding evolution coefficients also increases.

From Figure 4(d), it can be observed that different values of $j_{0}$ produce evolution coefficients that are able to correctly detect the evolution in the example data stream. The selection of a particular value for $j_{0}$ depends on the underlying distribution of the data, as well as the number of data items considered for the estimation, which is controlled by the size of the windows $w_{1}$ and $w_{2}$.

In order to provide a general guideline for the selection of $w_{1}$ and $w_{2}$, Figure 5 shows LECs calculated using different pairs of window sizes and different values for $j_{0}$ for four example data streams that present changes in mean at different times. The main observation from Figure 5 is that, to allow LECs to properly capture changes in the underlying generative process of the data, the number of data items covered by $w_{2}$ should be at least four times smaller than the number 


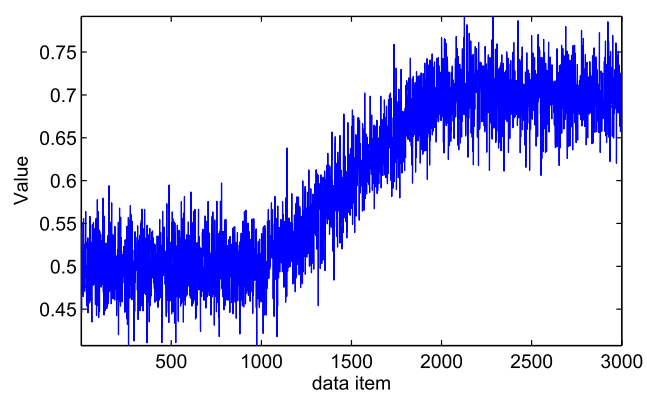

(a)

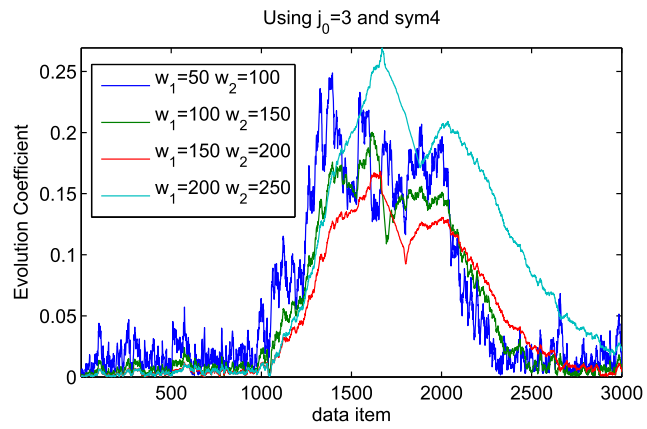

(c)

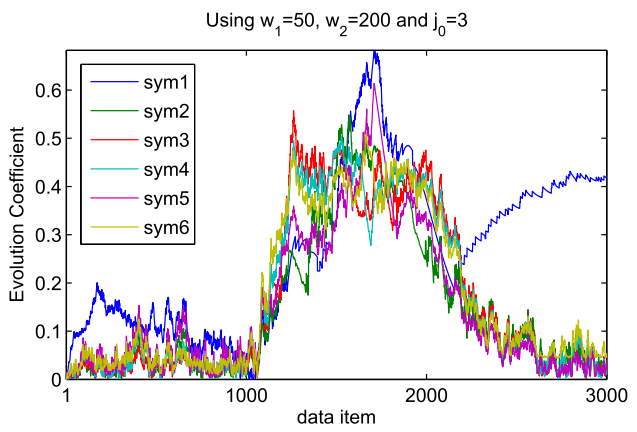

(e)

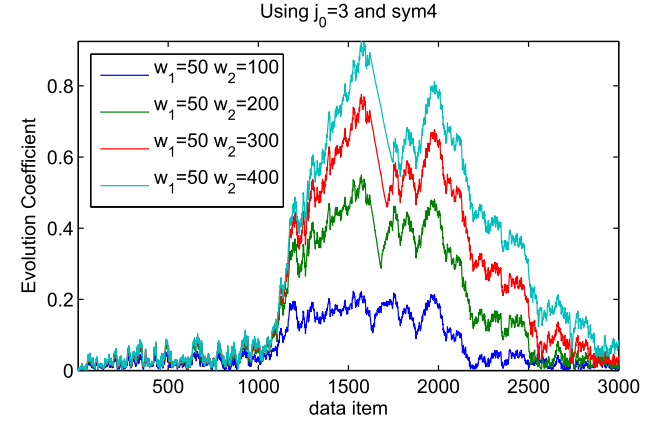

(b)

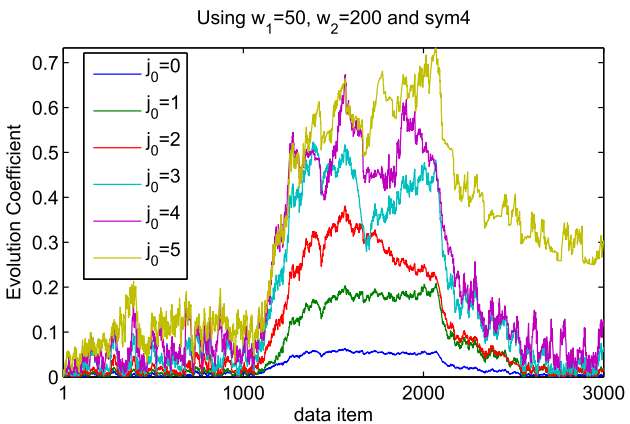

(d)

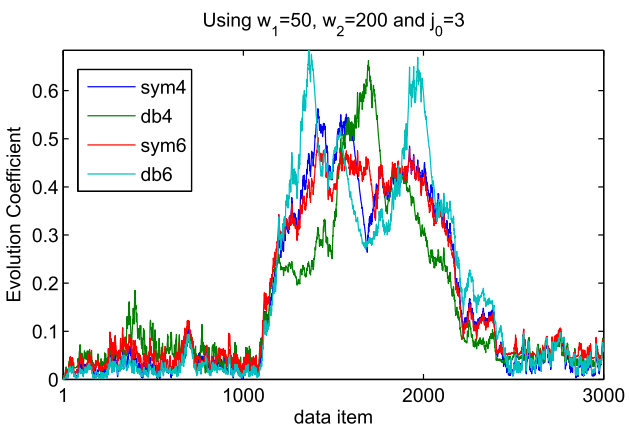

(f)

Fig. 4. Evolution coefficients for an example data stream (a) versus; (b) the difference $w_{2}-w_{1}$; (c) the pair of window sizes $w_{1}$ and $w_{2}$; (d) the initial scale $j_{0}$; (e) the order of the scaling function $N_{\phi_{j_{0}}}$; (f) the scaling function employed.

of data items between consecutive changes. Regarding the selection of $w_{1}$, we set $w_{1}=w_{2} / 2$ and, in this way we use a similar setting for the windows sizes as the benchmark approach (Aggarwal 2005). Regarding this setting, we can see that $w_{1}=125$ and $w_{2}=250$ is the only pair of windows that can correctly detect changes in the example data stream $d$ in Figure 5 for different values of $j_{0}$. The right column of Figure 5 shows the data stream $d$ whose second change occurs 1,000 data items after the first change.

Since the underlying distribution of data is unknown, for the selection of $j_{0}$ an approach similar to the one used to select the bandwidth parameter in KDE is proposed, that is to select $j_{0}$ 

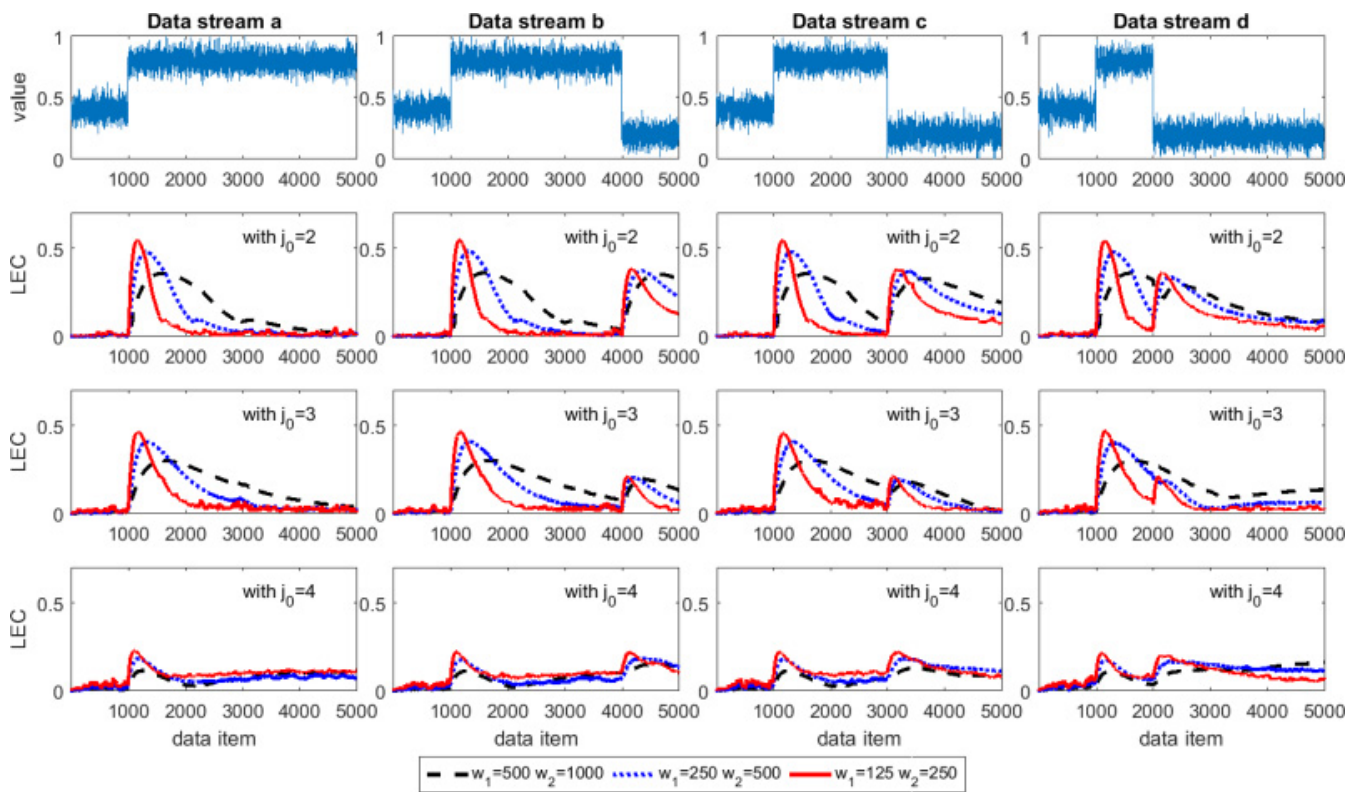

Fig. 5. Window size and the detection of changes.

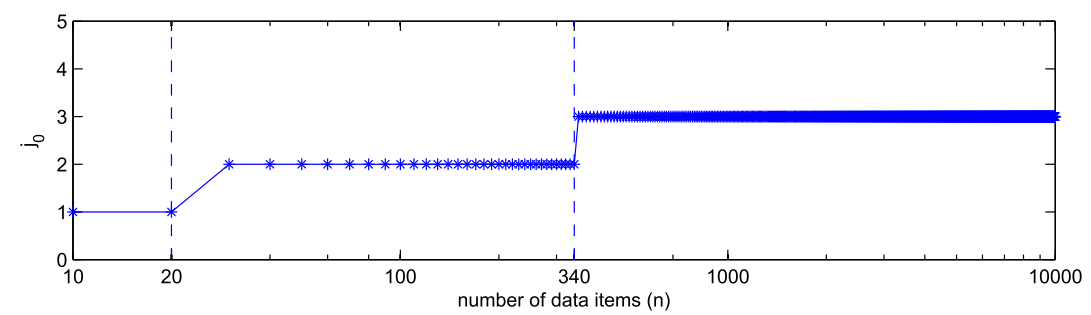

Fig. 6. Selection of $j_{0}$ for different values of $n$.

according to the optimal value for a normal distribution. We evaluate five different values $j_{0}$ for 500 values of $n$, ranging from 10 to 10,000 data items from a normal distribution $\mathcal{N}(0,1)$. Then an approximation of the Mean Integrated Squared Error (MISE) between the true density and their WDE estimates is obtained. For each value of $n$, the value of $j_{0}$ that reports the lowest MISE is selected as optimal. It is important to recall that since in the context of WDE and RWDE input data requires to be normalized between the range $[0,1]$ the variance of the data is not considered for the selection of $j_{0}$. Figure 6 shows the optimal values of $j_{0}$ for different values of $n$ determined according to the procedure above highlighted.

4.1.1 Estimation of Time Required to Calculate GECs. In this section, we evaluate the number of data items that can be processed per second ${ }^{3}$ for the estimation of GECs changing: (i) the order of the filter $N_{\phi_{0}}$, and (ii) the initial scale $j_{0}$. Note that since $N_{\phi_{0}}$ is similar for Daubechies and Symlets wavelet families, the reported results are valid for both wavelet families.

\footnotetext{
${ }^{3}$ The computer system used to generate the results reported in this section was an Intel i5 2500k with 16GB of RAM, running Linux Ubuntu 11.04 and the simulation environment was MATLAB R2010b.
} 
Table 3. Data Items per Second for the Estimation of GECs

\begin{tabular}{cccccccc}
\hline \multicolumn{6}{c}{$m$} & \multicolumn{7}{c}{ Order of the Filter $N_{\phi_{0}}$} \\
& $j_{0}$ & 1 & 2 & 3 & 4 & 5 & 6 \\
\hline \multirow{4}{*}{1} & 0 & 4,522 & 2,215 & 1,356 & 963 & 846 & 818 \\
& 1 & 4,437 & 2,180 & 1,361 & 959 & 843 & 808 \\
& 2 & 4,225 & 2,159 & 1,348 & 957 & 850 & 808 \\
& 3 & 3,961 & 2,086 & 1,313 & 952 & 842 & 807 \\
& 4 & 3,568 & 2,000 & 1,310 & 957 & 842 & 799 \\
& 5 & 3,317 & 1,984 & 1,299 & 945 & 818 & 800 \\
\hline \multirow{4}{*}{10} & 0 & 501 & 230 & 139 & 97 & 86 & 82 \\
& 1 & 482 & 229 & 139 & 97 & 85 & 81 \\
& 2 & 457 & 225 & 137 & 98 & 86 & 82 \\
& 3 & 427 & 216 & 134 & 97 & 85 & 81 \\
& 4 & 382 & 206 & 134 & 96 & 85 & 81 \\
& 5 & 353 & 204 & 133 & 96 & 85 & 79 \\
\hline \multirow{4}{*}{100} & 0 & 51 & 23 & 14 & 10 & 9 & 8 \\
& 1 & 49 & 23 & 14 & 10 & 9 & 8 \\
& 2 & 46 & 22 & 14 & 10 & 9 & 8 \\
& 3 & 43 & 22 & 13 & 10 & 8 & 8 \\
& 4 & 38 & 21 & 13 & 10 & 9 & 8 \\
& 5 & 36 & 21 & 13 & 10 & 8 & 8 \\
\hline
\end{tabular}

The first observation from Table 3 is that the number of data items that can be processed per second is inversely proportional to the order of the filter $N_{\phi_{0}}$. Also, a 100-dimensional data stream can update GECs about 10 times per second using any value for $N_{\phi_{0}}$. Hence, the real time estimation of GECs for high dimensional data is feasible with the proposed framework. Regarding the number of data items that can be processed per second when varying the scale $j_{0}$, it is not significantly affected for different values of $j_{0}$. The above results show that the proposed framework is a promising tool for the analysis of multidimensional data streams that can be implemented in personal computers with modest computational capabilities.

\subsection{Computational Complexity Assessment}

In this subsection, we empirically evaluate the complexity of the alternative velocity density frameworks in the construction of TVPs.

Two are the aspects included in the comparisons: the number of data items processed per second (i) as a function of the time window $h_{t}$, and (ii) as a function of the number of coordinates $\beta$ chosen for the evaluation of the velocity density. For this experiment, we use a synthetically generated two-dimensional data stream where each data item is drawn from a Gaussian distribution with means $\mu=[0.50 .5]$ and with covariance matrix $\Sigma=\sigma^{2} I_{2}$, where $\sigma^{2}=0.01$ and $I_{2}$ representing the two-dimensional identity matrix. In Figure 7, we show the corresponding results.

Note that, in Figure 7(a), only one curve is plotted for the proposed RWDE algorithm. This is because in the proposed approach the processing of a particular number of data items is independent of the value of $h_{t}$. In contrast, for the KDE-based method, different values of $h_{t}$ correspond to different processing times for a given number of data items. It is clear from Figure 7(a) that although for small values of $h_{t}$ the VDKDE framework requires less processing 


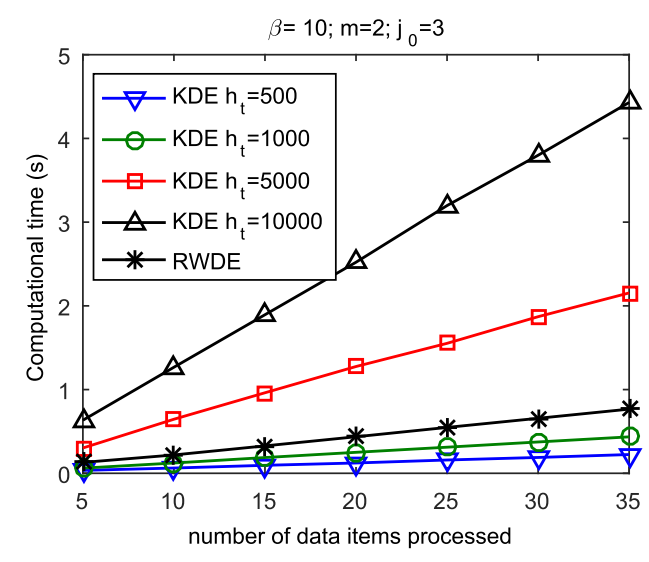

(a)

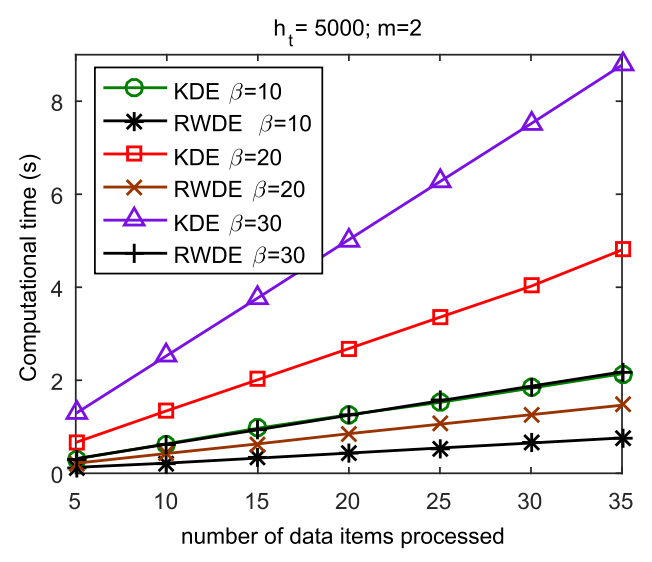

(b)

Fig. 7. Computational time versus number of data items processed per second: (a) for different values of $h_{t}$; (b) for different values of $\beta$.

time, the appeal of the proposed approach is that, in the construction of TVPs, the required processing time is independent of the time horizon of the analysis.

Regarding the number of coordinates $\beta$ selected at each dimension for the evaluation of TVPs, it can be observed from Figure 7(b) that, for similar values of $\beta$, TVPs obtained with the proposed framework have a reduced processing time.

\subsection{Example 1: Change Detection and Evolution Diagnosis}

We first evaluate the change detection performance of the proposed algorithm on two-dimensional synthetic data from a Gaussian mixture density. For comparison purpose, we also evaluate two recently published change detection algorithms: the semi-parametric log-likelihood criterion (SPLL) algorithm presented in Kuncheva (2013) and the relative density-ratio estimation (RuLSIF) algorithm presented in Liu et al. (2013). In the SPLL, two sliding windows of different lengths are implemented and an average squared Mahalanobis distance is considered for change detection criteria (i.e., as change point score); and in RuLSIF, a retrospective change point detection scheme is employed where change point is detected from relative density ratio estimation using Pearson divergence. For the proposed algorithm, we use GECs as the change point scores. The peaks of change point scores are defined as the detection alarms (DA) similar to Liu et al. (2013) and Desobry et al. (2005), and in order to reduce the noisy peaks in the data, we consider only those peaks which have a minimum peak prominence of 0.1 (10\% of maximum normalized change point score). A true positive (TP) is detected if there is (i) a DA and (ii) the detected alarm is the first detection within $N_{\text {min }}$ samples distance from actual change point $(C P)$. This $N_{\text {min }}$ samples delay corresponds to the minimum number of samples between any two change points in data. A DA is considered as a false positive $(F P)$ if it does not satisfy the conditions of true positive. True positive rate $(T P R)$ is defined as $T P R=\sum_{i} T P_{i} / \sum_{i} C P_{i}$ and false positive rate $(F P R)$ is defined as $F P R=\left(\sum_{i} D A_{i}-\sum_{i} T P_{i}\right) / \sum_{i} D A_{i}$

We generate a two-dimensional data stream from a mixture of Gaussian density that changes with time. The mixture has three Gaussian components and samples are generated from 1 to 35 "states or blocks" of these mixture coefficients. The values of these states of mixture coefficients $M_{1}, M_{2}$, and $M_{3}$ are shown in Table 4. This data stream contains a mixture of small and large changes in the underlying coefficients in consecutive states. Also for states $1-5$ and $13-18$ mixture 
Table 4. Mixture Coefficients for Evolving Data from a Gaussian Mixture Density

\begin{tabular}{c|ccc||c|ccc||c|ccc}
\hline$N$ & $M 1$ & $M 2$ & M3 & $N$ & $M 1$ & $M 2$ & M3 & $N$ & $M 1$ & $M 2$ & M3 \\
\hline 1 & 0.25 & 0.50 & 0.25 & 13 & 0.050 & 0.90 & 0.050 & 25 & 0.225 & 0.55 & 0.225 \\
2 & 0.25 & 0.50 & 0.25 & 14 & 0.050 & 0.90 & 0.050 & 26 & 0.250 & 0.50 & 0.250 \\
3 & 0.25 & 0.50 & 0.25 & 15 & 0.050 & 0.90 & 0.050 & 27 & 0.275 & 0.45 & 0.275 \\
4 & 0.25 & 0.50 & 0.25 & 16 & 0.050 & 0.90 & 0.050 & 28 & 0.300 & 0.40 & 0.300 \\
5 & 0.25 & 0.50 & 0.25 & 17 & 0.050 & 0.90 & 0.050 & 29 & 0.325 & 0.35 & 0.325 \\
6 & 0.30 & 0.40 & 0.30 & 18 & 0.050 & 0.90 & 0.050 & 30 & 0.350 & 0.30 & 0.350 \\
7 & 0.35 & 0.30 & 0.35 & 19 & 0.075 & 0.85 & 0.075 & 31 & 0.375 & 0.25 & 0.375 \\
8 & 0.40 & 0.20 & 0.40 & 20 & 0.100 & 0.80 & 0.100 & 32 & 0.400 & 0.20 & 0.400 \\
9 & 0.45 & 0.10 & 0.45 & 21 & 0.125 & 0.75 & 0.125 & 33 & 0.425 & 0.15 & 0.425 \\
10 & 0.35 & 0.30 & 0.35 & 22 & 0.150 & 0.70 & 0.105 & 34 & 0.450 & 0.10 & 0.450 \\
11 & 0.25 & 0.50 & 0.25 & 23 & 0.175 & 0.65 & 0.175 & 35 & 0.475 & 0.05 & 0.475 \\
12 & 0.15 & 0.70 & 0.15 & 24 & 0.200 & 0.60 & 0.200 & & & & \\
\hline
\end{tabular}

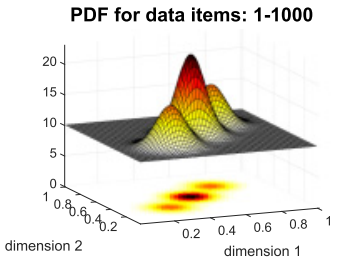

(a)

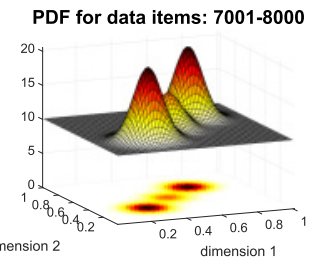

(b)

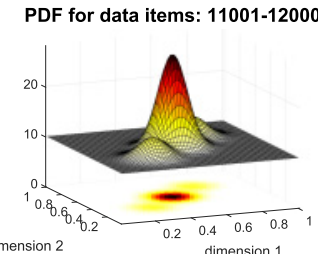

(c)

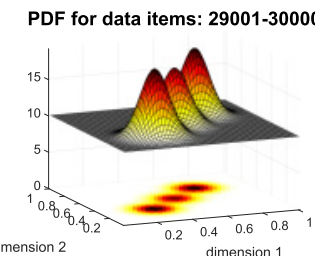

(d)

Fig. 8. PDF of Evolving Data-1000 at different time periods. (a) samples 1-1000, (b) samples 7001-8000, (c) samples 11001-12000, (d) samples 29001-30000.

values do not change, which means that data is being generated from the same density for longer period of time.

The two-dimensional mean and covariance matrix of each mixture component do not change with time and remains constant, and are given by

$$
\mathbf{U}=\left[\begin{array}{ll}
0.3 & 0.3 \\
0.5 & 0.5 \\
0.7 & 0.7
\end{array}\right], \quad \Sigma_{\mathbf{i}}=\left[\begin{array}{lr}
0.6 & 0 \\
0 & 0.6
\end{array}\right] \times 10^{-2}, \text { for } \quad i=1,2,3 .
$$

Two types of datasets are generated from these mixture of Gaussian densities that only differ in number of samples being generated from each of these 35 states: (1) Evolving data-500, where 500 samples are generated from each state and (2) Evolving data-1000, where 1,000 samples are generated from each state. Figure 8 shows, for evolving data-1000, the density function graphic representation for states $1,8,12$, and 30 .

Change detection performance in both datasets "Evolving data-500" and "Evolving data-1000" is shown in Figure 9(a) and (b) where a representative set of comparative results are presented. It can be seen from Figure 9(a) and (b) that the proposed GECs outperform RuLSIF and SPLL in change detection in multidimensional data where density function is complex and evolves at different rates in time. Note also that for the proposed algorithm, as the window $w_{1}$ size increases from $0.25 \times w_{2}$ to $0.50 \times w_{2}$, better TPR curve is obtained and, as expected, TPR curve obtained for data-1000 is better then data-500. However, gain in detection accuracy comes with an increased delay in detection of true positives due to larger window sizes. So, a trade-off between delay and detection accuracy will be required when selecting the window sizes. Figure 9 (c) shows the change 
(a) Evolving Data-500 with Minimum peak prominance 0.1

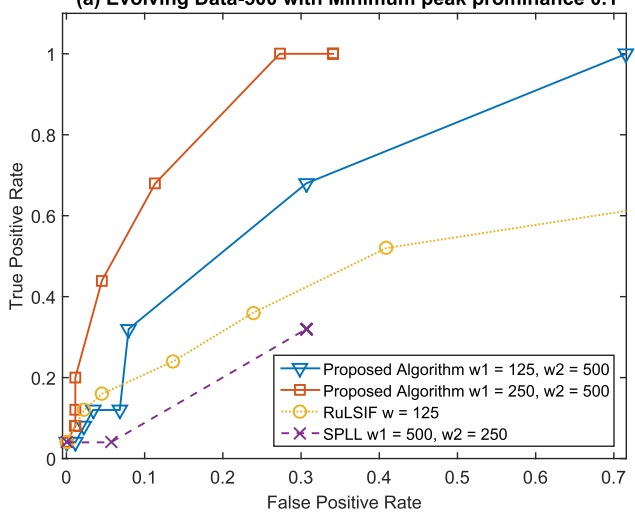

(a)

(b) Evolving Data-1000 with Minimum peak prominence 0.1

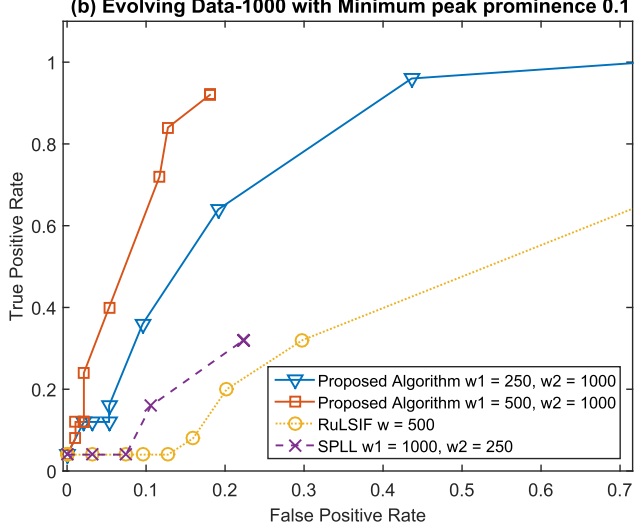

(b)

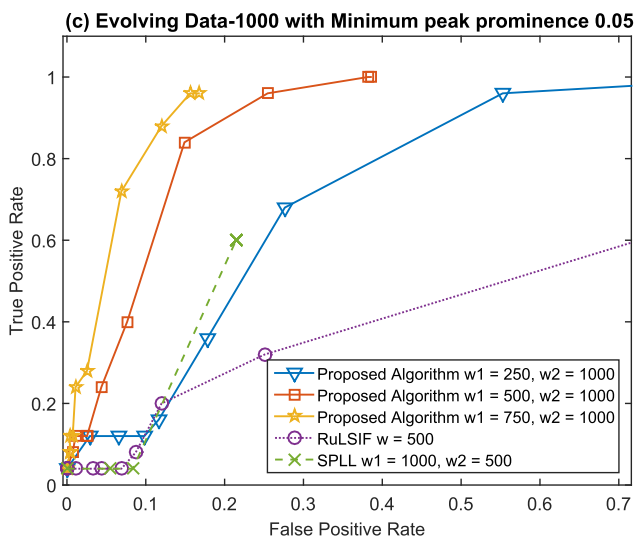

(c)

Fig. 9. Comparison of change detection with minimum peak prominence of 0.1 in Data-500 (a) and Data-100 (b); change detection with minimum peak prominence of 0.05 in Data-1000 (c). 
Table 5. Delay Comparison for Change Detection in Evolving Data from Gaussian Mixture Denstity

\begin{tabular}{c||c|c|c}
\hline Algorithm & $\begin{array}{c}\text { Figure 9(a) } \\
\text { \# of samples }\end{array}$ & $\begin{array}{c}\text { Figure 9(b) } \\
\text { \# of samples }\end{array}$ & $\begin{array}{c}\text { Figure 9(c) } \\
\text { \# of samples }\end{array}$ \\
\hline RuLSIF & 209 & 384 & 362 \\
\hline SPLL & 246 & 182 & 359 \\
\hline Proposed algorithm $w_{1}=0.25 \times w_{2}$ & 152 & 264 & 175 \\
\hline Proposed algorithm $w_{1}=0.50 \times w_{2}$ & 245 & 413 & 319 \\
\hline Proposed algorithm $w_{1}=0.75 \times w_{2}$ & \multicolumn{3}{|c}{} \\
\hline
\end{tabular}

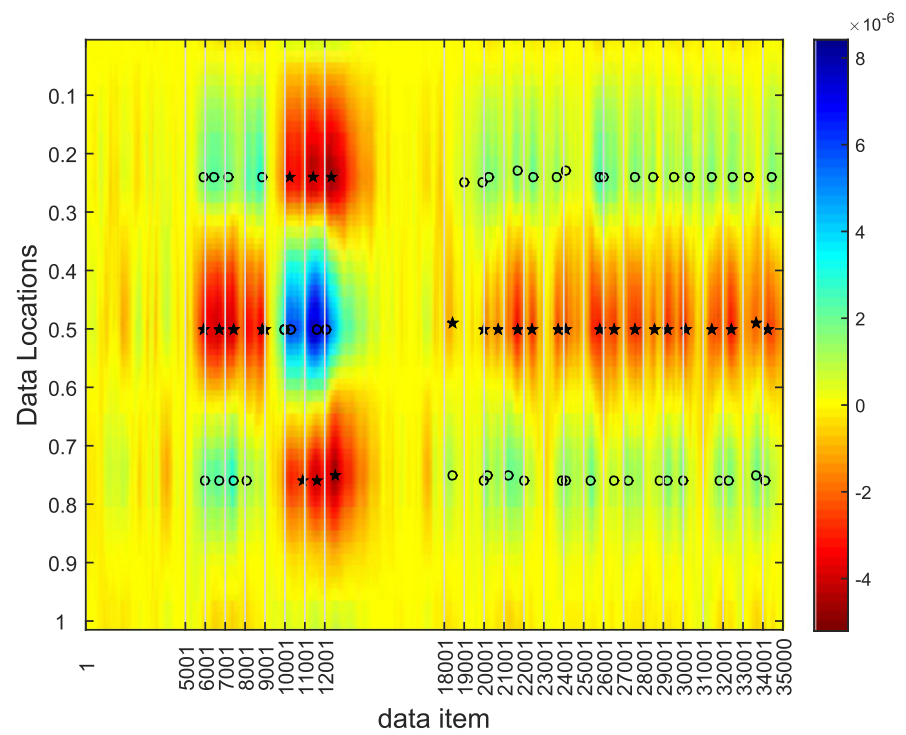

(a)

Fig. 10. Change characterization in the density of dimension- 1 of Evolving Data- 1000 with time, Using $w 1=$ 500 and $w 2=1,000$.

detection performance when we decrease the minimum peak prominence to 0.05 . As expected false positives will increase and curves will achieve TPR with higher FPR.

Average sample delays in detection for all algorithms in Figure 9 are presented in Table 5, where it can be seen that the proposed algorithm has sample delays of the same order of magnitude than the other algorithms and, in fact, it gives the lowest delay in case of curves in Figure 9(a) and 9(c). In the case of curves shown in Figure 9(b), SPLL gives the lowest delay but we note that its TPR is also very low.

4.3.1 Evolution Diagnosis. The proposed algorithm also provides additional insight into characteristics of data that cannot be obtained from the change detection algorithm RuLSIF (Liu et al. 2013) (as it directly optimize the density-ratio without estimating the densities) nor the SPLL algorithm (Kuncheva 2013) (because although in this algorithm density is estimated for $w_{1}$, a log-likelihood bound is used as a change measure). In Figure 10, the velocity density $V D D$ of dimension-1 of the "Evolving data-1000" data stream is shown (the VDD of dimension-2 has very similar characteristics), which presents the evolution or change in the density of data at different 
data locations over time. The darker shade at a particular data location means an increase in the density value and red shade represents a decrease in the density at that data location and intensity corresponds to the amount of change. We also note that, in Figure 10, the concept of data coagulation and data dissolution regions introduced in Aggarwal (2005) can be observed. It represents changes in the density of data at a time instant " $t$," which are in fact the increments and decrements in density at different data locations, respectively. The associated epicenters of data coagulation $(\circ)$ and data dissolution $(\star)$ are also shown in Figure 10, which conform with the changes in the states of mixture coefficients in Table 4.

\subsection{Example 2: Gas Sensor Array Under Dynamic Gas Mixtures}

In this real world example, we analyze the evolution of the measurements of a gas sensor array containing 16 chemical sensors reporting gas mixtures whose concentration levels vary over time. The aim of this experiment is to compare the evolution diagnosis capabilities of the proposed velocity approach with the one reported in Aggarwal (2005). For this purpose, we use the experimental dataset generated in Fonollosa et al. (2015) for the mixture composed of Ethylene and Methane on air. The dataset contains one time series for each of the 16 the chemical sensors in the array, so it can be considered a 16-dimensional dataset, where each time series contains 4,178,504 data points and was constructed by streaming the sensors' measurements during approximately 12 hours of uninterrupted data gathering. The concentration transitions were set at random times and to random concentration levels. The dataset includes the following transitions: increasing, decreasing, or setting to zero the concentration of one volatile, while the concentration of the other volatile is kept constant (either at a fixed or at zero concentration level).

In this experiment, the first million data points are used to obtain their corresponding evolution coefficients using VDKDE and the proposed method. Note that the original multidimensional VDKDE formulation cannot be directly applied here since, if we consider that to moderately characterize a given density we need at least 10 points distributed along the support of the density, then we will need to evaluate $10^{16}$ points for each data item processed. Hence, for benchmarking purposes, the evolution coefficients obtained using VDKDE are computed separately for each dimension. For VDKDE, we select a window size $h_{t}=1,000$ and bandwidth of the Gaussian Kernel $\sigma_{K D E}=0.06$, while for the proposed VDC we use a scale $j_{0}=3$ and the windows $w_{1}=500$ and $w_{2}=1,000 .{ }^{4}$ Results for this experiment are depicted in Figure 11, where the two upper plots (a, b) show the concentration levels over time of each of the two gases in the mixture, while the three pixel plots at the bottom (c, d, e) show the sensor array measurements and the evolution coefficients for the two evaluated methods. Note that in the three pixel plots the $y$-axis represents the sensor and the darker the color the higher the detected increase in the evolution coefficient.

The key observation from Figure 11 is that both methods are able to track the evolution of the measurements in the sensor array for the 16 sensors. Every time there is a change in the concentration of ethylene or methane there is an increase in the evolution coefficient reported by both velocity density approaches. In order to quantitatively asses how similar are results from both methods, we compute, for each sensor, the CC between the 1,000,000 evolution coefficients obtained using the VDKDE approach with the ones reported by the proposed VDC approach. The resulting CCs for each sensor are shown in Table 6. Using the guide to verbally describe statistical quantities (Evans 1996), results from Table 6 indicate that there is a very strong linear relationship between evolution coefficients reported by both velocity density methods in 12 out of 16 sensors (CC is higher than 0.8 ) and a strong correlation in the remaining 4 sensors. For the aggregate

\footnotetext{
${ }^{4}$ The values for $\sigma_{K D E}$ and $j_{0}$ were selected using a grid search to find the values that better characterize the changes in the dataset.
} 

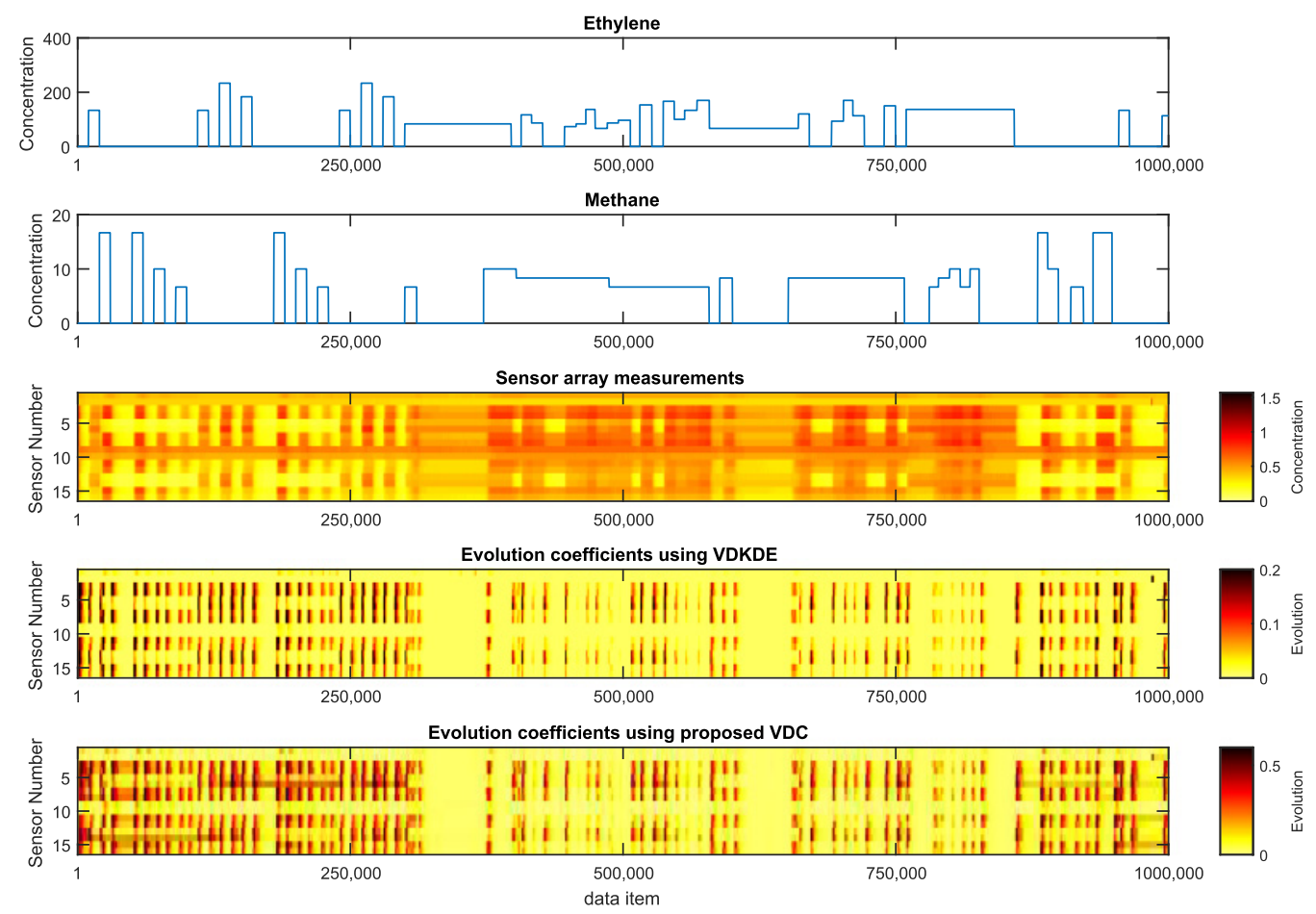

Fig. 11. Results for gas sensor array experiments.

Table 6. Correlation Between Evolution Coefficients Computed Using VDKDE and VDC

\begin{tabular}{cc|cc|cc|cc}
\hline Sensor & CC & Sensor & CC & Sensor & CC & Sensor & CC \\
\hline 1 & 0.64 & 5 & 0.85 & 9 & 0.79 & 13 & 0.86 \\
2 & 0.75 & 6 & 0.78 & 10 & 0.82 & 14 & 0.81 \\
3 & 0.84 & 7 & 0.83 & 11 & 0.85 & 15 & 0.82 \\
4 & 0.85 & 8 & 0.84 & 12 & 0.86 & 16 & 0.86 \\
\hline
\end{tabular}

results from all sensors, we compute the two-dimensional cross-correlation between the evolution coefficients for the 16 sensors, obtaining a value of 0.84 , which confirms the very strong linear relationship between the two paradigms to characterize evolution in data streams.

\subsection{Example 3: UK's Daily Temperature from 1960 to 2006}

The aim of this experiment is to exemplify how the proposed velocity density framework can be useful to perform more robust analysis in domains involving geospatial data. For this real world example, the proposed data stream evolution diagnosis framework is applied using the daily $5 \mathrm{~km} \times$ $5 \mathrm{~km}$ gridded temperature dataset for the UK from the UK's National Weather Service (Met Office 2012). We use the daily gridded dataset of mean temperatures (10,359 cells) for the period 1960 to 2006. Each cell consists of 17,167 values, where each value represents an estimate for the daily mean temperature of the center point of the $5 \times 5 \mathrm{~km}^{2}$ grid cell. Each time series cell is treated as a single data stream. 


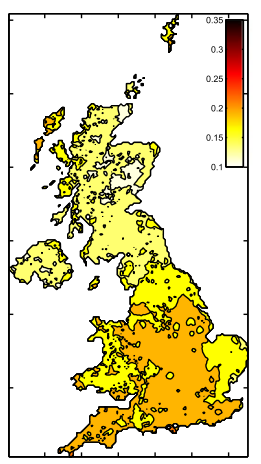

(a)

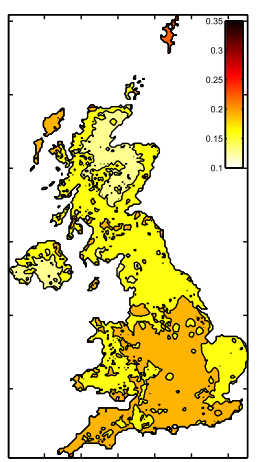

(b)

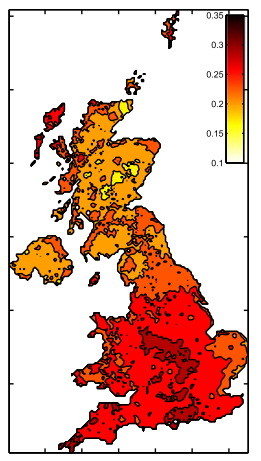

(c)

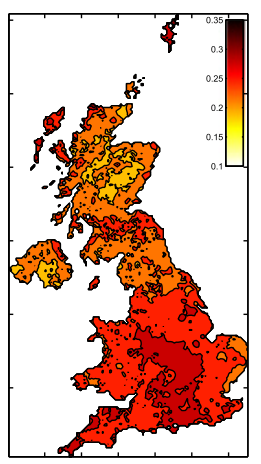

(d)

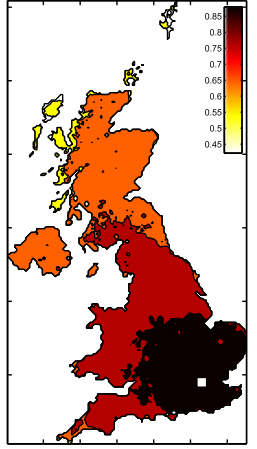

(e)

Fig. 12. Results for UK's temperature example. Averaged evolution coefficient for daily temperatures in the UK for year 2006, over 1 year (a) and over 5 years (b) using $w_{1}=365, w_{2}=1,825$; over 1 year (c) and over 5 years $(\mathrm{d})$ using $w_{1}=365, w_{2}=3,650$. (e) Correlation coefficient between evolution coefficients from 1960 to 2006 for Westminster (white square) and the rest of the locations in the UK using $w_{1}=365, w_{2}=1,825$, and $w_{\alpha}=3,600$.

This example consists in obtaining the VDC, and in turn the corresponding LEC at each point in the grid, for each of the 17,167 time stamps. Specifically, we evaluate two pairs of window sizes [ $w_{1}$ $w_{2}$ ]. The two pairs of windows consider the period of one year for $w_{1}$, that is $w_{1}=365$. Regarding the second window $w_{2}$, we use $w_{2}=(5)(365)=1,825$ and $w_{2}=(10)(365)=3,650$, corresponding to five years and ten years, respectively.

In Figure 12, we plot a map of the UK showing the average of evolution coefficients over 1 (Figure 12(a) and (c)) and 5 years (Figure 12(b) and (d)). We can observe that, in general, the southern part of the UK, the Western isles, the isles of Orkney (in the north and west of the UK), are the ones with the highest temperature evolution for the two time periods studied.

We also investigate the correlation between temperature evolution between the London borough of Westminster (white square in Figure 12(e)) and the rest of the Locations in the UK for the period 1960-2006. In this case, we compute, at every time stamp, the CC for the past 3,600 evolution coefficients between the cell corresponding to Westminster (Easting: 530,709 Northing: 179,631 ) and the remaining 10,358 cells in the grid. We use $w_{1}=365, w_{2}=1,825$, and $w_{\alpha}=3,600$. Then, we average the resulting 17,167 CCs at each location to produce the map depicted in Figure 12(e). As we would have expected for geospatial data, the temperature evolution is spatially correlated (higher correlation values are represented by dark shades). The cells associated to East of England and South East England are the regions that report the highest correlation of temperature evolution.

\subsection{Example 4: Hong Kong's Air Pollution Index for 2008-2013}

The aim of this experiment is to provide another example to illustrate how the diagnosis of data evolution can offer additional insights from data from different domains. This real world example considers the hourly Air Pollution Index (API) from Air Quality Monitoring Network of the Environmental Protection Department (EPD) Hong Kong (EPD 2015). In Figure 13(a), we show the location of the stations. Out of these 14 monitoring stations, 3 stations namely Causeway Bay, Central, and Mong Kok are roadside stations with monitoring station height less than 5 meters and the other stations are general stations with station height ranging from 11 to $27.5 \mathrm{~m}$. 


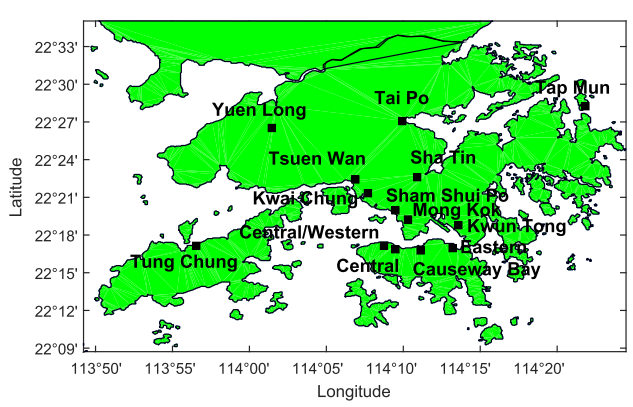

(a)

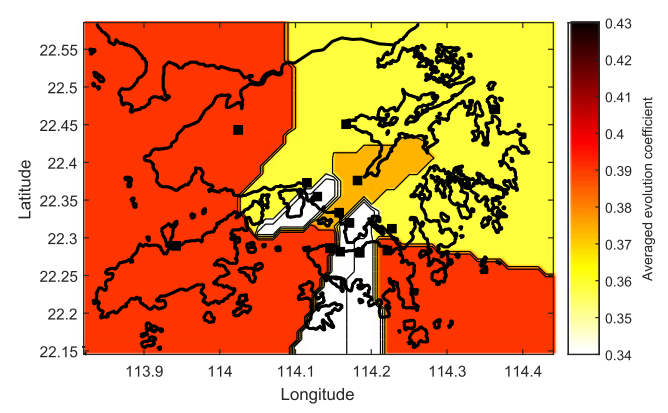

(b)

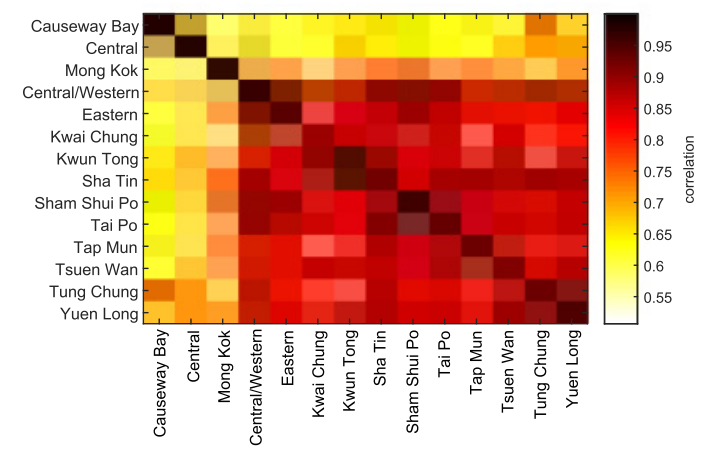

(c)

Fig. 13. Results for Hong Kong's pollution example. (a) Location of the monitoring stations; (b) averaged evolution coefficient using $w_{1}=720$ and $w_{2}=3,600$ for 2008-2013; (c) averaged correlation coefficients for evolution coefficients from different stations using $w_{\alpha}=3,600$.

The example consists in obtaining the LEC, for every hour between $01 / 01 / 2008$ and $29 / 12 / 2013$ for each of the stations in the network. Hence, we have 14 one-dimensional data streams of evolution coefficients with a total of 52,340 data items in each of them. We evaluate one pair of window sizes, while the first window considers the period of one month that is $w_{1}=(30)(24)=720$, the second window is set to cover the period of 5 months $w_{2}=(5)(30)(24)=3,600$. For visualization, we average all the evolution coefficients of each station and then apply a nearest neighbor interpolation to obtain a map showing the averaged evolution coefficient for the whole Hong Kong area for the period 2008-2013. From Figure 13(b), the areas covered by Yuen Long, Tung Chung, Central/Western, and Eastern stations are the ones that report, the highest API evolution. In contrast, Kwai Chung, Mong Kok, Central and Causeway Bay are the stations whose pollution levels present the lower evolution, which can be expected by the fact that pollution level remains the same in roadside stations where heavy traffic is always present.

To produce Figure 13(c), we obtain, at every time stamp, the CC for the past 8,640 evolution coefficients $\left(w_{\alpha}=8,640\right)$ between each of the 912 -combinations of the 14 stations. We obtain the resulting stream of 52,340 CCs that are averaged and use a pixel plot where each diagonal pixel, presents the correlation with itself.

Figure 13(c) is obtained using the EWMCC algorithm. It can be seen that the API evolution for Causeway Bay, Central and Mong Kok stations is the less correlated with the API evolution of the remaining stations. This can be expected as these stations monitor air pollution only at $5 \mathrm{~m}$ height 
on roads surrounded by concentrated high-rise buildings, where heavy traffic is constantly present (EPD 2015).

\section{FINAL REMARKS}

In this article, we propose a novel multidimensional data stream evolution diagnosis framework which extends the velocity density concept (Aggarwal 2005) using RWDE. The proposed framework, which is a novel tool for analyzing multidimensional data streams, has the following key advantages: (1) it requires constant memory independent of the amount of data to be analyzed; (2) the computation complexity is significantly lower than existing approaches, (3) the diagnosis of data evolution is performed with only one pass of the data. The proposed framework presents a considerable reduction in computational time and in memory requirements when compared to the method proposed in Aggarwal (2005). Furthermore, the capability of estimating both, velocity densities and evolution coefficients separately to each dimension, makes the proposed algorithm more versatile than to its KDE-based counterpart since, it allows the application of the framework in a wider range of domains. With the help of four examples, the potential of the proposed framework is highlighted. While some examples compare the proposed framework against the KDE-based approach and against some change detection methods, other examples present some guidelines for its application with geospatial data. In particular, the example that compares the proposed framework in the context of a change detection problem, shows very encouraging results. Further work will consider combining the results in this article with our recently published work on time series descriptors (García-Treviño and Barria 2014).

\section{REFERENCES}

Charu C. Aggarwal. 2005. On change diagnosis in evolving data streams. IEEE Transactions on Knowledge and Data Engineering 17, 5 (2005), 587-600.

Charu C. Aggarwal. 2007. An introduction to data streams. In Data Streams (The Kluwer International Series on Advances in Database Systems), Charu C. Aggarwal and Ahmed K. Elmagarmid (Eds.). Vol. 31, Springer, 1-8.

Charu C. Aggarwal and Philip S. Yu. 2007. On string classification in data streams. In Proceedings of the 13th ACM SIGKDD International Conference on Knowledge discovery and data mining (KDD'07). ACM, New York, NY, 36-45.

Feng Cao, Martin Estert, Weining Qian, and Aoying Zhou. 2006. Density-based clustering over an evolving data stream with noise. In Proceedings of the 2006 SIAM International Conference on Data Mining. SIAM, 328-339.

Kyle A. Caudle and Edward Wegman. 2009. Nonparametric density estimation of streaming data using orthogonal series. Computational Statistics \& Data Analysis 53, 12 (2009), 3980-3986.

N. N. Céncov. 1962. Evaluation of an unknown distribution density from observations. Soviet Mathematics Doklady 3 (1962), $1559-1562$.

Yixin Chen and Li Tu. 2007. Density-based clustering for real-time stream data. In Proceedings of the 13th ACM SIGKDD International Conference on Knowledge Discovery and Data Mining. ACM, 133-142.

Graham Cormode and Minos Garofalakis. 2007. Sketching probabilistic data streams. In Proceedings of the 2007 ACM SIGMOD International Conference on Management of Data (SIGMOD'07). ACM, New York, NY, 281-292.

Graham Cormode and Marios Hadjieleftheriou. 2009. Finding the frequent items in streams of data. Communications ACM 52, 10 (Oct. 2009), 97-105.

Bi-Ru Dai, Jen-Wei Huang, Mi-Yen Yeh, and Ming-Syan Chen. 2006. Adaptive clustering for multiple evolving streams. IEEE Transactions on Knowledge and Data Engineering 18, 9 (Sept. 2006), 1166-1180.

Frédéric Desobry, Manuel Davy, and Christian Doncarli. 2005. An online kernel change detection algorithm. IEEE Transactions on Signal Processing 53, 8 (2005), 2961-2974.

EPD. 2015. Environmental Protection Department (EPD) Hong Kong. Retrieved December, 2015 from http://www.epd-asg. gov.hk/.

James D. Evans. 1996. Straightforward Statistics for the Behavioral Sciences. Brooks/Cole.

Christos Faloutsos. 2004. Indexing and mining streams. In Proceedings of the 2004 ACM SIGMOD International Conference on Management of data (SIGMOD’04). ACM, New York, NY, 969-969.

Jordi Fonollosa, Sadique Sheik, Ramón Huerta, and Santiago Marco. 2015. Reservoir computing compensates slow response of chemosensor arrays exposed to fast varying gas concentrations in continuous monitoring. Sensors and Actuators B: Chemical 215 (2015), 618-629. 
Edgar S. García-Treviño and Javier A. Barria. 2012. Online wavelet-based density estimation for non-stationary streaming data. Computational Statistics and Data Analysis 56, 2 (Feb. 2012), 327-344.

Edgar S. García-Treviño and Javier A. Barria. 2014. Structural generative descriptions for time series classification. IEEE Transactions on Cybernetics 44, 10 (Oct. 2014), 1978-1991. DOI : http://dx.doi.org/10.1109/TCYB.2014.2322310

Vivekanand Gopalkrishnan. 2008. Querying time-series streams. In Proceedings of the 11th International Conference on Extending database technology: Advances in database technology (EDBT'08). ACM, New York, NY, 547-558.

Philipp Kranen, Ira Assent, Corinna Baldauf, and Thomas Seidl. 2011. The ClusTree: Indexing micro-clusters for anytime stream mining. Knowledge and Information Systems 29, 2 (2011), 249-272.

Ludmila I. Kuncheva. 2013. Change detection in streaming multivariate data using likelihood detectors. IEEE Transactions on Knowledge and Data Engineering 25, 5 (2013), 1175-1180.

Song Liu, Makoto Yamada, Nigel Collier, and Masashi Sugiyama. 2013. Change-point detection in time-series data by relative density-ratio estimation. Neural Networks 43 (2013), 72-83.

Met Office. 2012. UK's National Weather Service. October, 2012 from http://www.metoffice.gov.uk/.

R. T. Ogden. 1997. Essential Wavelets for Statistical Applications and Data Analysis. Birkhauser.

Márcia Oliveira and João Gama. 2012. A framework to monitor clusters evolution applied to economy and finance problems. Intelligent Data Analysis 16, 1 (2012), 93-111.

Pedro Pereira Rodrigues, Joao Gama, and Joao Pedro Pedroso. 2008. Hierarchical clustering of time-series data streams. IEEE Transactions on Knowledge and Data Engineering 20 (2008), 615-627.

Jonathan A. Silva, Elaine R. Faria, Rodrigo C. Barros, Eduardo R. Hruschka, André CPLF de Carvalho, and João Gama. 2013. Data stream clustering: A survey. ACM Computing Surveys 46, 1 (2013), 13.

Myra Spiliopoulou, Irene Ntoutsi, Yannis Theodoridis, and Rene Schult. 2006. Monic: Modeling and monitoring cluster transitions. In Proceedings of the 12th ACM SIGKDD International Conference on Knowledge Discovery and Data Mining. ACM, 706-711.

Brani Vidakovic. 1999. Statistical Modeling by Wavelets. Wiley, New York.

Haixun Wang, Wei Fan, Philip S. Yu, and Jiawei Han. 2003. Mining concept-drifting data streams using ensemble classifiers. In Proceedings of the 9th ACM SIGKDD International Conference on Knowledge Discovery and Data Mining. ACM, 226-235.

Min Wang and X. Wang. 2003. Efficient evaluation of composite correlations for streaming time series. In Advances in WebAge Information Management, Guozhu Dong, Changjie Tang, and Wei Wang (Eds.), Lecture Notes in Computer Science, vol. 2762. Springer, Berlin, 369-380.

Ying Yang, Xindong Wu, and Xingquan Zhu. 2006. Mining in anticipation for concept change: Proactive-reactive prediction in data streams. Data Mining and Knowledge Discovery 13, 3 (2006), 261-289.

Received May 2016; revised May 2017; accepted June 2017 